\title{
छs \\ Origin of gate hysteresis in p-type Si-doped AlGaAs/GaAs heterostructures
}

\author{
A. M. Burke, ${ }^{1}$ D. E. J. Waddington, ${ }^{1}$ D. J. Carrad, ${ }^{1}$ R. W. Lyttleton, ${ }^{1}$ H. H. Tan, ${ }^{2}$ P. J. Reece, ${ }^{1}$ O. Klochan, ${ }^{1}$ A. R. Hamilton, ${ }^{1}$ \\ A. Rai, ${ }^{3}$ D. Reuter, ${ }^{3}$ A. D. Wieck, ${ }^{3}$ and A. P. Micolich ${ }^{1, *}$ \\ ${ }^{1}$ School of Physics, University of New South Wales, Sydney NSW 2052, Australia \\ ${ }^{2}$ Department of Electronic Materials Engineering, Research School of Physics and Engineering, The Australian National University, \\ Canberra ACT 0200, Australia \\ ${ }^{3}$ Angewandte Festkörperphysik, Ruhr-Universität Bochum, D-44780 Bochum, Germany
}

(Received 12 July 2012; published 8 October 2012)

\begin{abstract}
Gate instability/hysteresis in modulation-doped $p$-type AlGaAs/GaAs heterostructures impedes the development of nanoscale hole devices, which are of interest for topics from quantum computing to novel spin physics. We present an extended study conducted using custom-grown, matched modulation-doped $n$-type and $p$-type heterostructures, with and without insulated gates, aimed at understanding the origin of the hysteresis. We show the hysteresis is not due to the inherent "leakiness" of gates on $p$-type heterostructures, as commonly believed. Instead, hysteresis arises from a combination of GaAs surface-state trapping and charge migration in the doping layer. Our results provide insights into the physics of $\mathrm{Si}$ acceptors in $\mathrm{AlGaAs} / \mathrm{GaAs}$ heterostructures, including widely debated acceptor complexes such as $\mathrm{Si}-\mathrm{X}$. We propose methods for mitigating the gate hysteresis, including poisoning the modulation-doping layer with deep-trapping centers (e.g., by codoping with transition metal species) and replacing the Schottky gates with degenerately doped semiconductor gates to screen the conducting channel from GaAs surface states.
\end{abstract}

DOI: 10.1103/PhysRevB.86.165309

PACS number(s): 71.55.Eq, 72.20.-i, 73.20.At, 77.55.dj

\section{INTRODUCTION}

The modulation-doped AlGaAs/GaAs heterostructure is a materials platform of great importance to the study of nanoscale electronic devices with quantum mechanical functionalities and their development towards future technologies. ${ }^{1,2}$ Studies of the two-dimensional electron gas (2DEG) formed in an AlGaAs/GaAs heterostructure at temperatures below $4 \mathrm{~K}$ have met with great success, both in terms of the novel physics of $2 \mathrm{D}$ electrons $\mathrm{s}^{3,4}$ and as an underpinning technology for quantum wires, ${ }^{5,6}$ quantum dots, ${ }^{7-9}$ and other ballistic transport devices. ${ }^{2}$ Devices based on two-dimensional hole gases (2DHGs) have received less attention; this is not from a lack of interesting physics. The higher effective mass of holes leads to stronger carrier interactions, making 2DHGs of interest for studies of the metal-insulator transition ${ }^{10,11}$ and bilayer quantum Hall effect. ${ }^{12,13}$ Additionally, the spin- $\frac{3}{2}$ nature of holes, arising from strong spin-orbit interactions, has driven interest in novel phenomena in 2DHGs, such as $g$-factor anisotropy ${ }^{14}$ and anomalous spin-polarization,,${ }^{15}$ as well as studies of the 0.7 plateau in quantum point contacts (QPCs), ${ }^{16}$ the quantum dot Kondo effect ${ }^{17}$ and Berry's phase in Aharonov-Bohm rings. ${ }^{18}$ The reduced hyperfine interaction for holes in $\mathrm{GaAs}^{19}$ leads to reduced spin-decoherence time compared to electrons, ${ }^{20,21}$ making GaAs hole quantum dots of interest for quantum computing. ${ }^{22}$

Studies of low-dimensional hole devices are impeded by difficulties in making devices with high electronic stability and low noise/drift under electrostatic gating. Telegraph noise, instability, and gate hysteresis were particularly problematic in initial attempts to realize hole $\mathrm{QPCs}^{23-25}$ using Si-doped (311)A-oriented 2DHGs. Similar issues were reported for gated 2DHGs in C-doped (100) $\mathrm{AlGaAs} / \mathrm{GaAs}$ heterostructures. ${ }^{26-28}$ Numerous explanations have been offered, including surface diffusion of ohmic contact metal producing a low mobility layer at or close to the surface, ${ }^{29}$ charge transfer to states either at the interfaces or in the semiconductor, ${ }^{30}$ carrier trapping in deep acceptor levels or in insulating parallel doping layers, ${ }^{28}$ or that metallic gates on p-GaAs are "inherently leaky" because of a reduced Schottky barrier relative to $\mathrm{n}-\mathrm{GaAs} .{ }^{26,27,31}$ Firmly establishing the origin of the gate instability/hysteresis will contribute towards the development of improved materials and devices for the study of low-dimensional hole systems.

Here we show that gate instability/hysteresis in $p$-type modulation-doped $\mathrm{AlGaAs} / \mathrm{GaAs}$ heterostructures is caused by a complex interplay between surface-state trapping and gate-induced charge migration within the doping layer. We focus here on Si-doped (311)A-oriented heterostructures, but similar physics may occur in C-doped (100)-oriented heterostructures also. We use three different experimental approaches: First, we rule out direct charge leakage between the gate and semiconductor by studying devices where an insulator layer is deposited underneath the gates; two insulators, $\mathrm{Al}_{2} \mathrm{O}_{3}$ and polyimide, were investigated. We find that insulating the gates does not eliminate the hysteresis; instead, it makes the hysteresis significantly worse. A recent study of a QPC featuring $\mathrm{HfO}_{2}$-insulated gates on a Cdoped (100) AlGaAs/GaAs heterostructure ${ }^{31}$ also shows that hysteresis can remain despite insulating the gates. In our experiment, the semiconductor surface/gate interface is the only aspect that differs between the insulated and uninsulated gate devices; they are on the same heterostructure with the same doping. The resulting large difference in hysteresis points to surface states as an important contributing factor. Hence, our second approach was to investigate surface effects by studying matched $\mathrm{AlGaAs} / \mathrm{GaAs}$ heterostructures, both with and without an $\mathrm{Al}_{2} \mathrm{O}_{3}$ gate insulator layer, and Schottky-gated $p$-type heterostructures with $\left(\mathrm{NH}_{4}\right)_{2} \mathrm{~S}_{x}$ surface passivation treatment ${ }^{32-34}$ performed prior to gate deposition. In contrast to hole devices, the addition of the insulator does not induce 
hysteresis for electron devices but alters the pinch-off voltage. Sulfur passivation does not bring consistent improvement in $p$ type devices, despite increasing the photoluminescence yield on (100) and (311) surfaces. ${ }^{35}$ In the one instance where passivation did bring significant improvement, hysteresis was still observed. This led us to suspect charge migration in the doping layer. Hence, our third approach was to investigate dopant effects through variable temperature studies. In particular, we compare the hysteresis in hole devices to electron devices at elevated temperatures $T \sim 130 \mathrm{~K}$, where deep donors known as DX centers ${ }^{36}$ begin to detrap, ${ }^{37}$ allowing charge to migrate between Si dopant sites. We observe gate hysteresis in electron devices at $130 \mathrm{~K}$ very similar to that in hole devices at $T<4 \mathrm{~K}$. We also show that the hysteresis can be reduced in hole devices by reducing the thickness of the dopant layer from $80 \mathrm{~nm}$ to less than $5 \mathrm{~nm}$ ( $\delta$ doped). Overall, our results point to competition between surface-state and dopant-related processes with different time/energy scales as the cause of the hysteresis and have wider implications, given recent interest in surface charge as a source of scattering ${ }^{38}$ and dopant charge migration as a source of noise $\mathrm{e}^{39}$ in $\mathrm{AlGaAs} / \mathrm{GaAs}$ heterostructures and quantum devices.

The paper is structured as follows. Section II briefly addresses materials and methods, with an extended discussion presented in Appendix A. In Sec. III we focus on gate leakage and discuss hole devices featuring $\mathrm{Al}_{2} \mathrm{O}_{3}$ and polyimide gate insulators. Sections IV and V concentrate on surface states and charge migration in the dopant layer, respectively. Finally, in Sec. VI, we draw conclusions based on the overall results and discuss the broader implications of the work for the study of low-dimensional devices in AlGaAs/GaAs heterostructures. Appendices B-D contain additional supporting data.

\section{MATERIALS AND METHODS}

Five separate AlGaAs/GaAs heterostructures denoted 1-e, 1-h, 2-e, 2-h, and 3-h were made for this study; the first four are "matched" electron (-e) and hole (-h) heterostructures produced by deposition onto side-by-side halves of 2-inchdiameter GaAs substrate, one (100) oriented and the other (311) oriented. This ability to produced matched electron and hole heterostructures relies on the orientation-dependent amphoteric nature of $\mathrm{Si}$ dopants in AlGaAs. ${ }^{40}$ The first four wafers feature an $80 \mathrm{~nm}$ Si-doped AlGaAs modulation doping layer separated from the 2DHG/2DEG by $35 \mathrm{~nm}$ of undoped AlGaAs, and the fifth features a Si-doping layer separated from the 2DHG/2DEG by $21 \mathrm{~nm}$ of undoped AlGaAs. These heterostructures have typical carrier densities of $\sim 1.3 \times 10^{11} \mathrm{~cm}^{-2}$ and mobilities of $500000 \mathrm{~cm}^{2} / \mathrm{Vs}$ at temperature $T=300 \mathrm{mK}$. Devices were produced by standard GaAs device processing methods and involved definition of a Hall bar structure by wet etching, deposition and annealing of ohmic contacts, and deposition of Ti/Au gates and interconnects. $\mathrm{Al}_{2} \mathrm{O}_{3}$-insulated gate samples feature a $20 \mathrm{~nm} \mathrm{Al}{ }_{2} \mathrm{O}_{3}$ layer deposited using atomic layer deposition at $200{ }^{\circ} \mathrm{C}$ after Hall bar definition, with access for ohmic contacts provided using a buffered HF etch. The polyimide insulated gate sample was produced using photoprocessable polyimide (HD Microsystems) deposited after the ohmic contact anneal. Sulfur passivation was performed immediately before gate deposition by $2 \mathrm{~min}$ immersion in a $0.5 \%$ dilution of stock $\left(\mathrm{NH}_{4}\right)_{2} \mathrm{~S}_{x}$ solution prepared by adding $9.62 \mathrm{~g}$ of elemental sulfur (Aldrich) to $100 \mathrm{~mL}$ of $20 \%\left(\mathrm{NH}_{4}\right)_{2} \mathrm{~S}$ solution (Aldrich). The passivation is performed at $40{ }^{\circ} \mathrm{C}$ and care is taken to minimize air exposure between passivation and gate/interconnect deposition. Electrical measurements at $T \geqslant 4 \mathrm{~K}$ and $T=0.25-4 \mathrm{~K}$ were obtained using a liquid helium dip station and an Oxford Instruments Heliox ${ }^{3} \mathrm{He}$ cryostat, respectively. Standard two- and four-terminal ac lock-in techniques were used to measure the conducting channel's drain current $I_{d}$, typically with a $100 \mu \mathrm{V}$ constant voltage excitation at $73.3 \mathrm{~Hz}$ applied to the source. The gate bias $V_{g}$ was applied using a Keithley 2400 source-measure unit enabling continuous measurement of gate leakage current $I_{g}$ down to $100 \mathrm{pA}$. More complete details of heterostructure growth, device fabrication, and electrical measurement are given in Appendix A.

\section{GATE LEAKAGE AND INSULATED GATE DEVICE ON $p$-TYPE HETEROSTRUCTURES}

\section{A. Are Schottky-gates on $p$-type heterostructures really inherently leaky?}

A common explanation for instability and gate hysteresis in Schottky-gated $p$-type $\mathrm{AlGaAs} / \mathrm{GaAs}$ heterostructures is charge leakage from the metal surface gate into the heterostructure when the gate is positively biased to deplete the 2DHG. This often relies on the argument that Schottky gates on $p$-type heterostructures are inherently more leaky than on $n$-type heterostructures. ${ }^{26,27,31,41}$ Reference 27 suggests this occurs because (a) GaAs surface states cause the surface Fermi level to be pinned slightly closer to the valence band than the conduction band, making the Schottky barrier lower for $p$-type heterostructures, ${ }^{42}$ and (b) depletion of the hole gas requires the Schottky gates to be forward biased. The latter argument is incorrect-depletion is a reverse bias process whether the underlying structure is $n$-type or $p$-type; the bias convention is reversed under an inversion in doping type. ${ }^{43}$ The former argument requires caution for two reasons. First, in most heterostructures, the GaAs cap is intrinsic, and the dopants in the heterostructure, whether $n$ - or $p$-type, are in the AlGaAs layers more than $5 \mathrm{~nm}$ beneath the surface. Hence, the Schottky barrier is the same for either doping type, suppressing any relative difference in gate leakage. Second, Schottky barrier measurements are usually performed at $T=300 \mathrm{~K}$, where thermionic emission dominates any leakage current, ${ }^{42,43}$ whereas most quantum devices ${ }^{1-18,20-31,38,39}$ are studied at $T=4 \mathrm{~K}$, where thermionic emission is quenched due to its exponential temperature dependence. This should further suppress the leakage current difference between Schottky gates on $n$ - and $p$-type heterostructures.

Thus, metal gates on $p$ - and $n$-type heterostructures should behave similarly at low temperature aside from a sign reversal in bias convention (forward/reverse). To confirm this, Fig. 1(a) shows the gate leakage current $I_{g}$ vs gate bias $V_{g}$ for a Schottky-gated $p$-type heterostructure (these data are presented on a linear $I_{g}$ axis for comparison in Appendix B). The gate begins to pass significant current under forward 

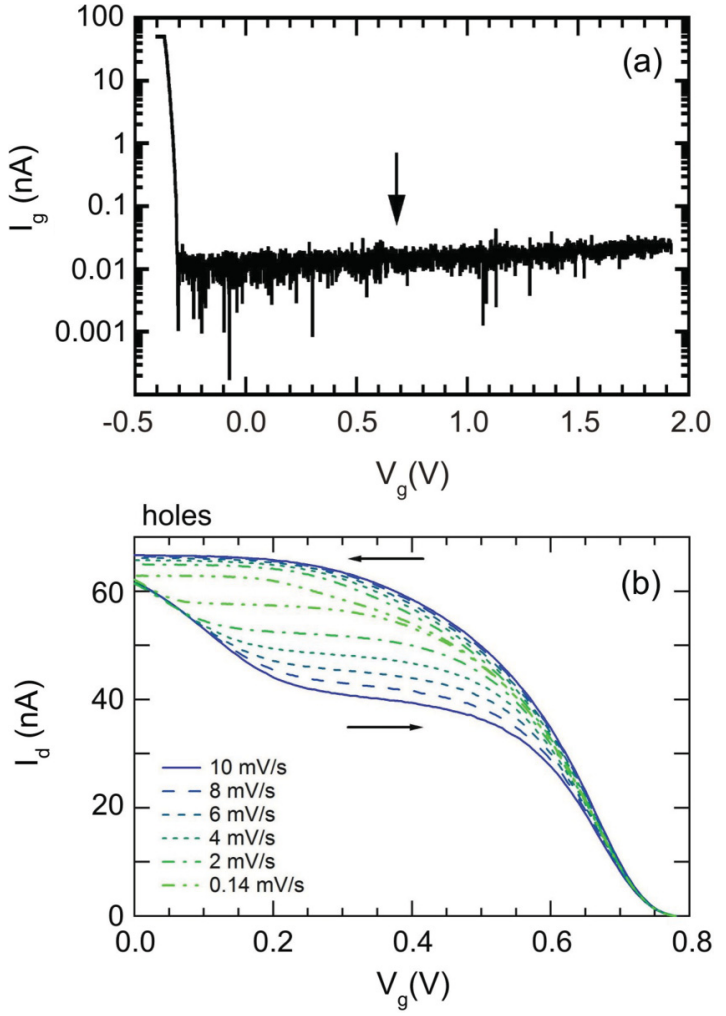

FIG. 1. (Color online) (a) Gate leakage current $I_{g}$ on a log-axis vs gate bias $V_{g}$ for a Schottky-gated modulation-doped $p$-type heterostructure. At positive $V_{g}, I_{g}$ remains less than $50 \mathrm{pA}$ up to $V_{g}=+2 \mathrm{~V}$, sufficient to achieve pinch-off for all uninsulated gate devices studied. In an $n$-type heterostructure, gate leakage would normally occur at $V_{g}=+0.68 \mathrm{~V}$ (indicated by the arrow) and is suppressed for negative $V_{g}$. (b) Channel current $I_{d}$ vs gate voltage $V_{g}$ at six different gate sweep rates for Device A. The horizontal arrows indicate the direction of travel around the hysteresis loop.

bias conditions at $V_{g} \approx-0.3 \mathrm{~V}$. Under reverse bias, where 2DHG depletion occurs, the leakage current gradually rises as $V_{g}$ is made increasingly positive, with $I_{g}<50 \mathrm{pA}$ for the entire range $0<V_{g}<+2 \mathrm{~V}$; this is sufficient to achieve pinch-off in all of our uninsulated devices. In contrast, a negative $V_{g}$, i.e., forward bias, produces strong gate leakage at $V_{g} \approx-0.3 \mathrm{~V}$. The misconception that Schottky gates on $p$-type $\mathrm{AlGaAs} / \mathrm{GaAs}$ heterostructures leak at positive $V_{g}$ may arise from the knowledge that Schottky gates on $n$-type AlGaAs/GaAs heterostructures leak for positive $V_{g} \gtrsim+0.68 \mathrm{~V}$ [indicated by the arrow in Fig. 1(a)]. Reverse bias leakage for $n$-type heterostructures usually does not occur until well beyond $V_{g}=-2 \mathrm{~V}$, consistent with the reverse bias behavior for the $p$-type heterostructure in Fig. 1(a). Despite this, Schottky gates on $n$-type AlGaAs/GaAs heterostructures leak relatively tiny amounts of charge under normal operation. ${ }^{44}$ This was demonstrated by Pioro-Ladrière et al., ${ }^{45}$ using a device consisting of a small quantum dot, isolated from the adjacent 2DEG, and coupled to a quantum point contact (QPC) charge sensor (see Fig. 7 of Ref. 45). The QPC detects charge leaking from the Schottky gates forming the dot, with extremely small leakage currents $\sim 10^{-20}$ A observed. These tiny currents are often associated with charge noise in $n$-type heterostructures ${ }^{39,44,45}$ but are not commonly known to produce strong gate hysteresis in $n$-type heterostructures to the level seen in $p$-type devices; the gates in electron devices are generally very stable (see Sec. IV A). Given this, it is not possible using Fig. 1(a) and the earlier discussion alone to definitively rule out gate leakage as a possible cause of gate hysteresis/instability in $p$-type heterostructures. The ultimate test, insulating the gates from the heterostructure, will be presented in Sec. III C; first, we characterize the hysteresis we observe in Schottky-gated 2DHGs.

\section{B. Hysteresis in a Schottky-gated p-type heterostructure}

Device A has Ti/Au gates deposited directly on the surface of 1-h. Figure 1(b) shows the measured drain current $I_{d}$ vs gate voltage $V_{g}$ from Device A at six $V_{g}$ sweep rates between 0 and $10 \mathrm{mV} / \mathrm{s}$. Hysteresis occurs for all $V_{g}$ but is most prominent for $0.1<V_{g}<0.5 \mathrm{~V}$. Note that $I_{d}$ reflects the channel conductivity, which can vary due to changes in either carrier density or mobility. Hall measurements with a small perpendicular magnetic field applied vs $V_{g}$ show a qualitatively identical hysteresis to that in Fig. 1(b), confirming that changes in $I_{d}$ with $V_{g}$ are predominantly density related. The direction of travel around the hysteresis loop [indicated by horizontal arrows in Fig. 1(b)] provides important clues about the origin of the hysteresis. First, it allows the most obvious cause of apparent hysteresis, recording delay in the measurement apparatus (sweep lag), to be ruled out. Any delay on the upsweep to positive $V_{g}$ causes a given depletion to occur at a higher apparent $V_{g}$, and, on the downsweep to $V_{g}=0$, reaccumulation of carriers occurs at a lower apparent $V_{g}$. The net result is a clockwise hysteresis loop-the direction of travel in Fig. 1(b) is clearly counterclockwise. Another key characteristic of sweep lag is that the hysteresis is strongest where the derivative $d I_{d} / d V_{g}$ is greatest. In Fig. 1(b) the hysteresis is strongest where $d I_{d} / d V_{g}$ is the smallest (see Appendix C). These two observations confirm that the hysteresis is not an instrumental issue; it instead originates within the device.

The effect of gate leakage depends on where the charge leaks to. In an entirely dc measurement, charge leaking directly to the $2 \mathrm{DHG}$ would add/subtract from the channel current. This would modify the $I_{d}-V_{g}$ characteristics but should not produce hysteresis. Here we measure $I_{d}$ using an ac lock-in technique; any gate leakage direct to the $2 \mathrm{DHG}$ does not appear in the measured $I_{d}$ vs $V_{g}$ unless it is an ac current at the reference frequency. An alternative is that charge leaks into trap states between the gate and $2 \mathrm{DHG}$; this will produce hysteresis if the trap time is not very small. Because a positive $V_{g}$ is applied to the gates to deplete the $2 \mathrm{DHG}$, any charge leaking from the gate will be positive, and its closer proximity to the $2 \mathrm{DHG}$ will increase the depletion at a given $V_{g}$ on the downsweep. Similarly, for a 2DEG the leaked charge will be negative, and it will also increase depletion; this is what produces the downward steps in the downsweep arm of the gate characteristics in Fig. 7(a) of Ref. 45. Returning our attention to holes, on the upsweep, reaccumulation in the $2 \mathrm{DHG}$ will be delayed by the need for the trapped positive charge to dissipate. This produces a clockwise hysteresis loop for holes, opposite to that in Fig. 1(b) and consistent with our argument in Sec. III $\mathrm{C}$ below that gate leakage does not cause the hysteresis. Note 
that gate leakage corresponds to a counterclockwise hysteresis loop for electrons due to the hysteresis loop being reflected about $V_{g}=0$ for carrier sign inversion; this is exactly the loop direction obtained in Fig. 7 of Ref. 45 where gate leakage does cause the hysteresis. The steps in Ref. 45 arise from the high sensitivity of their measurement configuration, single/few trap resolution will not be observed in the large area gate devices studied here. Note that the hysteresis loop for the hole QPC in Ref. 31 is counterclockwise, opposite the direction expected for hysteresis due to gate leakage.

We will present a possible explanation for the counterclockwise loop direction in Sec. III D, but, first, we continue towards ruling out gate leakage as the cause of the hysteresis by looking at insulated-gate devices.

\section{Hysteresis in an $\mathrm{Al}_{2} \mathrm{O}_{3}$ insulated-gate $p$-type heterostructure}

Figures 2(a) and 2(b) show $I_{d}$ and $I_{g}$ vs $V_{g}$ for Device B, with $\mathrm{Ti} / \mathrm{Au}$ gates insulated by a $20 \mathrm{~nm} \mathrm{Al}{ }_{2} \mathrm{O}_{3}$ layer. We would expect hysteresis to be heavily suppressed in this device if it is caused by gate leakage. Instead, the hysteresis is enhanced, and we need to drive $V_{g}$ to $\mathrm{Al}_{2} \mathrm{O}_{3}$ insulator breakdown to even approach pinch-off. A higher pinch-off voltage is expected; Device B has increased gate-2DHG separation and an extra dielectric layer. We find a conversion factor $V_{g}^{\text {Ins }}=1.21 V_{g}$ relating the insulated and uninsulated gate biases $V_{g}^{\text {Ins }}$ and $V_{g}$ using a parallel-plate capacitor model, assuming GaAs, AlGaAs, and $\mathrm{Al}_{2} \mathrm{O}_{3}$ dielectric constants of 12.9, 12.0, and 9.3, respectively. It is clear from Figs. 1(b) and 2(a) that the shift in pinch-off bias from $+0.77 \mathrm{~V}$ to $+5.8 \mathrm{~V}$, i.e., by a factor of 7.53, far exceeds that expected from simply adding $20 \mathrm{~nm}$ of $\mathrm{Al}_{2} \mathrm{O}_{3}$. To confirm that Device B's large pinch-off voltage is not a fabrication problem, we measured a second device differing only in top-gate pattern/area (see Appendix D). This

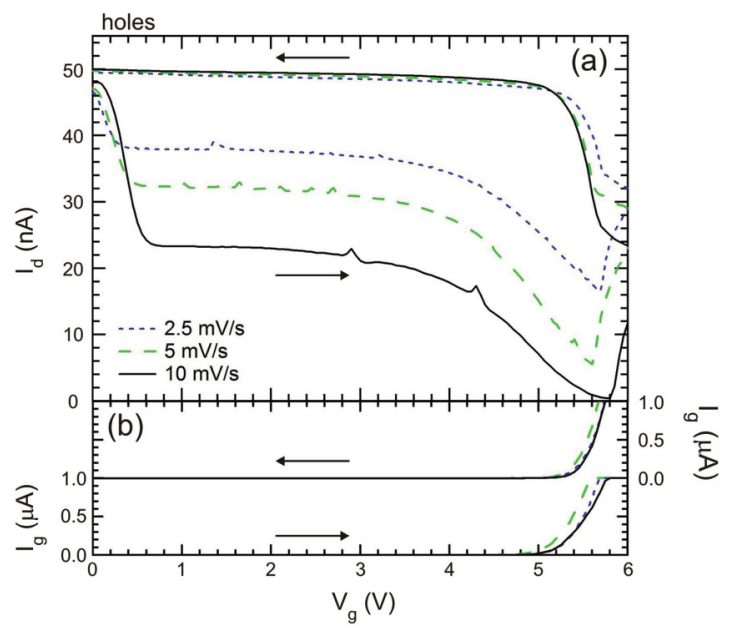

FIG. 2. (Color online) (a) Channel current $I_{d}$ and (b) gate leakage current $I_{g}$ vs gate voltage $V_{g}$ at three different gate sweep rates for Device $\mathrm{B}\left(20 \mathrm{~nm} \mathrm{Al}_{2} \mathrm{O}_{3}\right.$ layer on heterostructure 1-h). Horizontal arrows indicate sweep direction. In (b) the left/right axis and lower/upper data are for the up/down sweep, respectively. Once $I_{g}$ reaches $1 \mu \mathrm{A}$ the gate voltage source implements current limiting, holding $V_{g}$ fixed. Hence, for $V_{g} \gtrsim+5.6 \mathrm{~V}$ the data in (a) should be considered as $I_{d}$ vs time $t$ at fixed $V_{g}$, with each $0.2 \mathrm{~V}$ minor tick in $V_{g}$ corresponding to 80,40 , and $20 \mathrm{~s}$ for sweep rates of $2.5,5$, and $10 \mathrm{mV} / \mathrm{s}$, respectively. gave similar behavior, with a large $V_{g} \sim+5 \mathrm{~V}$ required to achieve even modest depletion $\left(\sim 22 \%\right.$ reduction in $\left.I_{d}\right)$.

The features in Figs. 1(b) and 2(a) are qualitatively identical, but we highlight two places where the quantitative differences are substantial. First, the current plateau at intermediate $V_{g}$ is much longer for Device $\mathrm{B}$, delaying pinch-off accordingly. This creates the illusion that the initial drop in $I_{d}$ in the upsweep is steeper; the opposite is true with the initial drop in Fig. 1(b) complete by $V_{g}=+0.1-+0.2 \mathrm{~V}$, whereas the same drop in $I_{d}$ takes $V_{g}=+0.4-+0.6 \mathrm{~V}$ in Fig. 2(a). Second, there is a distinct asymmetry in the sweep rate dependence of upsweeps and downsweeps: the upsweep path depends heavily on sweep rate while the downsweep path is largely independent of sweep rate. This is also evident in Fig. 1(b) but is heavily exacerbated by the $\mathrm{Al}_{2} \mathrm{O}_{3}$ layer.

The insulator-semiconductor interface can have a radical effect on performance in devices with a shallow conducting channel. ${ }^{38,46-48}$ Oxide insulators are particularly troublesome due to high interface trap densities; improvement is often obtained using polymeric insulators e.g., polyimide. ${ }^{49}$ To explore this, we studied Device $\mathrm{C}$, which features gates insulated with $140 \mathrm{~nm}$ of polyimide. This gave qualitatively similar results to Device B (see Appendix D). Pinch-off cannot be achieved in Device $C$ either, largely due to the much lower breakdown voltage $V_{g} \gtrsim+2.8 \mathrm{~V}$ for the polyimide layer. At breakdown, $I_{d}$ has only fallen by $\sim 20 \%$ of its $V_{g}=0$ value.

\section{Possible explanations for this form of hysteresis}

A possible explanation for a counterclockwise hysteresis loop and its particular shape in Fig. 1(b) is the gradual population/depopulation of a layer of net negative charge between the gate and 2DHG (N.B. the charge on the gate is positive, and this cannot be gate leakage). On the upsweep, the positive $V_{g}$ places positive charge on the gate, and this ideally results in depletion of the $2 \mathrm{DHG}$ (i.e., reduced carrier density). But, if adding positive charge to the gate instead results in net negative charge accumulation between gate and 2DHG, then depletion stalls, producing a reduced transconductance $\left|d I_{d} / d V_{g}\right|$ and perhaps even an $I_{d}$ plateau. The link between a plateau in $I_{d}$ versus $V_{g}$ and stalling of 2DHG depletion was confirmed by a corresponding plateau in Hall measurements of the 2DHG. If the capacity for net negative charge accumulation is finite, depletion eventually resumes, leading to pinch-off $\left(I_{d}=0\right)$. On the downsweep, positive charge is gradually removed from the gate, leading to $2 \mathrm{DHG}$ repopulation and loss of the accumulated net negative charge. If the net negative charge is held in deep-trapping sites, its loss may be very slow. Repopulation of the $2 \mathrm{DHG}$ will occur first, producing a rapid rise in $I_{d}$ to a level close to its initial value at $V_{g}=0$, followed by a long current plateau extending to $V_{g}=0$ as the net negative charge is lost.

An immediately obvious mechanism is charge trapping by surface states; it is well known in both oxide-insulated III-V FETs ${ }^{46,47}$ and organic semiconductor FETs. ${ }^{48}$ Another possible mechanism is charge migration in the modulationdoping layer. Here the use of "net" charge accumulation is deliberate and important. Silicon donors in the $\mathrm{Al}_{x} \mathrm{Ga}_{1-x} \mathrm{As}$ modulation doping layer of an $n$-type heterostructure can take two different configurations: a shallow hydrogenic donor 
occupying a $\mathrm{Ga}$ site or, for $x>0.2$, a metastable donor where the $\mathrm{Si}$ atom is displaced into an interstitial position along the $\langle 111\rangle$ direction by lattice distortion. ${ }^{50}$ The DX centers act as deep traps; when the device is cooled below $\sim 120 \mathrm{~K}$, the DX centers capture free electrons in the doping layer to become $\mathrm{DX}^{-}$, locking some fraction of the remaining hydrogenic donors in a positive charge state. This "freezing" of dopant layer charge is vital to the stability and reproducibility of the electronic properties of devices based on Si-doped $n$-type AlGaAs/GaAs heterostructures at low temperatures. ${ }^{37,51,52}$ Comparatively little is known about the defect physics of Si dopants in (311)-oriented p-type $\mathrm{AlGaAs} / \mathrm{GaAs}$ heterostructures. In addition to acting as a simple substitutional acceptor by occupying an As site, $\mathrm{Si}$ has also been suggested to form an acceptor complex/deep trap known as $\mathrm{Si}-\mathrm{X} .^{53}$ Initial studies ${ }^{53}$ suggested that $\mathrm{Si}-\mathrm{X}$ consisted of a $\mathrm{Si}_{\mathrm{As}}$ acceptor adjacent to a $\mathrm{Ga}$ site vacancy $\mathrm{V}_{\mathrm{Ga}}$ (subscript denotes site occupied). Raman and IR spectroscopy regarding the existence of $\mathrm{Si}-\mathrm{X}$ in heavily $\mathrm{Si}$-doped (311)A GaAs layers is controversial, with data suggesting that it does $^{54}$ and does not ${ }^{55}$ exist. Further work suggested modified structures for $\mathrm{Si}-\mathrm{X}$, first as a $\mathrm{V}_{\mathrm{Ga}}-\mathrm{Si}_{\mathrm{As}}-\mathrm{As}_{\mathrm{Ga}}$ complex, ${ }^{56,57}$ but later ruled out in favor of a perturbed $\mathrm{Si}_{\mathrm{Ga}}-\mathrm{V}_{\mathrm{Ga}}$ center. ${ }^{58,59}$ These studies were all for GaAs; the existence and properties of Si-X-like complexes in $\mathrm{Al}_{0.33} \mathrm{Ga}_{0.67} \mathrm{As}$ are unknown. We comment further on possibilities for $\mathrm{Si}$ acceptor complexes and deep traps in Sec. VI.

Returning to hysteresis mechanisms, if the dopant layer traps are shallow or thermal energy is sufficient for a high detrapping rate, charge can migrate in response to the balance of charge between the gate and $2 \mathrm{DHG}$. For example, when $V_{g}=0$ it is energetically more favorable for the $2 \mathrm{DHG}$ side of the doping layer to be net negative and the gate side net positive. At pinch-off, the positively charged gate and depleted 2DHG favor the opposite (2DHG side net positive/gate side net negative). This gate-induced "tidal flow" of doping layer charge is equivalent to negative charge accumulation between gate and 2DHG towards generating a counterclockwise hysteresis loop, as demonstrated in Sec. V.

\section{THE INFLUENCE OF THE HETEROSTRUCTURE SURFACE}

\section{A. Hysteresis in Schottky and insulated gate devices on matched $\boldsymbol{n}$-type heterostructures}

To better understand the comparative behavior of Devices $\mathrm{A}$ and $\mathrm{B}$, we prepared Devices $\mathrm{D}$ and $\mathrm{E}$ on 1-e: Device D has no $\mathrm{Al}_{2} \mathrm{O}_{3}$ layer and Device $\mathrm{E}$ has a $20 \mathrm{~nm} \mathrm{Al}_{2} \mathrm{O}_{3}$ layer grown simultaneously with that in Device B. Figures 3(a) and 3(b) present the electrical characteristics for Devices D and E. In each case, the apparent hysteresis is minimal and the loop direction is consistent with sweep lag (N.B. reflection of gate characteristics about $V_{g}=0$ due to sign-inversion reverses loop direction; for electrons sweep lag goes counterclockwise). On its own, the lack of device-induced hysteresis in Devices D and $E$ does not allow us to pinpoint the origin of the hysteresis solely to surface states or charge migration, as the shift from 1-h to 1-e entails a change in both the dopant physics and the surface orientation. While the (100) GaAs surface consists only
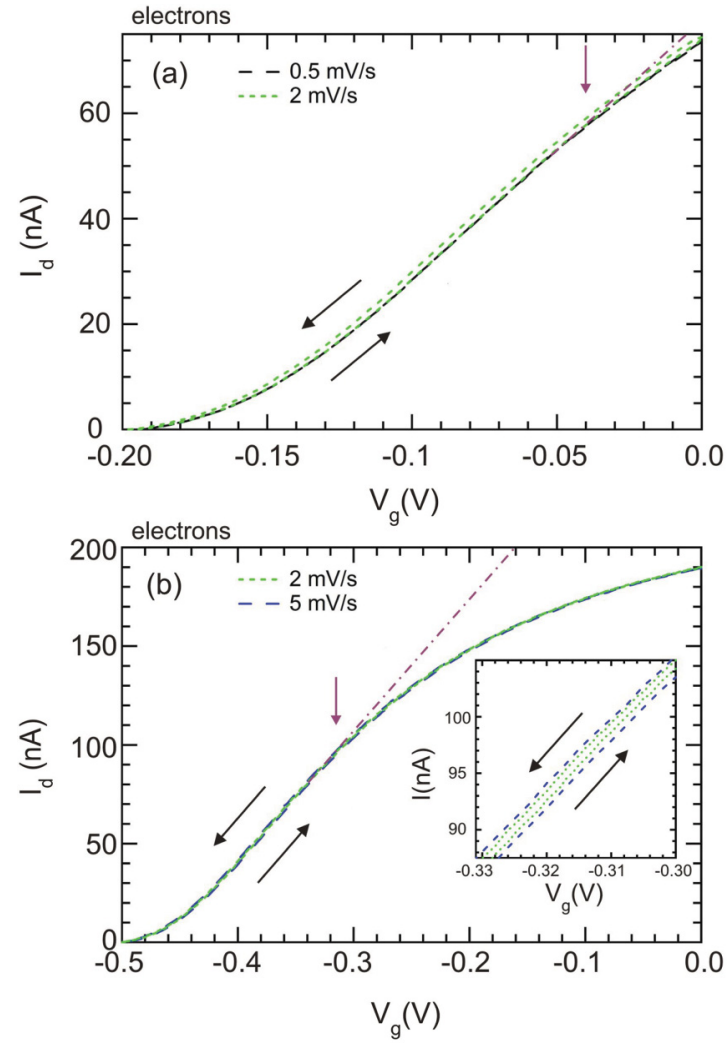

FIG. 3. (Color online) Channel current $I_{d}$ vs gate voltage $V_{g}$ for devices (a) without (Device D) and (b) with a $20 \mathrm{~nm} \mathrm{Al}_{2} \mathrm{O}_{3}$ gate insulator (Device E) on heterostructure 1-e. The inset to (b) shows a close-up of the data in the main panel to highlight the hysteresis. The arrows indicate hysteresis loop direction. The purple dot-dashed lines are guides to the eye highlighting the low-bias nonlinearity.

of double-dangling bonds, the (311) surface contains equal densities of single- and double-dangling bonds. ${ }^{60}$

Figure 3 has two notable features: The first is the pinch-off bias, which increases from $-0.20 \mathrm{~V}$ to $-0.50 \mathrm{~V}$ on addition of the $\mathrm{Al}_{2} \mathrm{O}_{3}$ layer. This $2.5 \times$ increase in pinch-off bias is less than the $7.53 \times$ found for holes but more than double the $1.21 \times$ expected from a parallel-plate capacitor model. The second feature is the distinct nonlinearity in $I_{d}$ versus $V_{g}$ at low bias in Device E. Although this low bias behavior in Fig. 3(b) differs in appearance from that in Fig. 2(a), in both cases it represents a reduced depletion rate for a given change in gate bias and may have a similar origin. A comparison of Figs. 3(a) and 3(b) suggests the low-bias nonlinearity arises from the $\mathrm{Al}_{2} \mathrm{O}_{3}$ layer; we believe it is caused by competition between the filling of surface states and 2DEG depletion. This hypothesis is further supported by extrapolating the linear trend at moderate $V_{g}$ to lower $V_{g}$, as per the purple dot-dash lines in Figs. 3(a) and 3(b). The vertical purple arrows indicate where the lowbias nonlinearity ends; $0.16 \mathrm{~V}$ and $0.184 \mathrm{~V}$ to the right of pinch-off in Figs. 3(a) and 3(b), respectively. The latter is $1.15 \times$ the former, very close to the $1.21 \times$ expected with the $\mathrm{Al}_{2} \mathrm{O}_{3}$ layer. The 5\% difference between the actual value of $0.184 \mathrm{~V}$ for Device $\mathrm{E}$ and the expected value of $0.194 \mathrm{~V}$ is well explained by the $5 \%$ difference in measured $V_{g}=0$ carrier density between Devices D and E. The findings above 
suggest that most $2 \mathrm{DEG}$ depletion occurs in the linear region in Figs. 3(a) and 3(b), with surface-state filling dominant for $V_{g} \gtrsim$ $-0.04 \mathrm{~V}$ in Device D and $V_{g} \gtrsim-0.32 \mathrm{~V}$ in Device E. Another way to envision this is as a threshold voltage shift induced by the surface states, as per oxide-insulated III-V FETs ${ }^{46}$ and organic semiconductor FETs. ${ }^{48}$ A final point of note is that the data in Fig. 3(a) are not linear all the way to $V_{g}=0$. This suggests that surface states have a measurable impact on Schottky-gated devices also. This is not surprising; one naturally expects a finite surface-state density for uninsulated GaAs surfaces.

\section{B. Sulfur passivation of Schottky-gated $p$-type heterostructures}

One approach to reducing the surface-state density is chemical passivation, the aim being to remove the native oxide and covalently satisfy all of the Ga and As dangling bonds. This ideally shifts the surface states out of the band gap and into the valence or conduction bands. ${ }^{33,61}$ Passivation is commonly achieved using aqueous and alcoholic chalcogenide solutions, particularly those containing sulfur, e.g., $\mathrm{Na}_{2} \mathrm{~S}^{32}$ or $\left(\mathrm{NH}_{4}\right)_{2} \mathrm{~S}^{33}$ A comprehensive review of chemical passivation of III-V surfaces is provided by Lebedev. ${ }^{34}$

The best approach to sulfur passivation involves difficult decisions among competing benefits. For example, while $\mathrm{Na}_{2} \mathrm{~S}$ treatment produces a surface passivation that is more robust to light/oxygen than $\left(\mathrm{NH}_{4}\right)_{2} \mathrm{~S}$ treatment, ${ }^{62}$ the latter produces surfaces with less $\mathrm{O}$, more $\mathrm{S}$, and no traces of $\mathrm{Na}{ }^{63}$ Alcoholic solutions are more effective than aqueous solutions, ${ }^{64}$ but alcoholic solutions are incompatible with photolithography resist, making them difficult it implement with patterned gates. ${ }^{35}$ The passivation treatment used here results from an extended study of different approaches to sulfur passivation of patterned-gate hole devices that will be reported elsewhere. ${ }^{35}$

Figures 4(a) and 4(b) show gate hysteresis data from passivated devices on 1-h (Devices F and G). We usually obtain data like those in Fig. 4(a) when including sulfur passivation in the fabrication process; pinch-off voltages between +0.57 and $+0.92 \mathrm{~V}$ are typically obtained depending on the treatment formulation. ${ }^{35}$ The Device G data in Fig. 4(b) represent an isolated instance where elimination of the current plateaus and a much lower pinch-off voltage $+0.27 \mathrm{~V}$ was observed. Note that a small counterclockwise hysteresis loop remains, consistent with that obtained when we examine the hysteresis generated by charge reorganization in the dopant layer in Sec. V. It is also consistent with the hysteresis we observe at $T<300 \mathrm{mK}$ and the data in Ref. 31. Caution is needed with Fig. 4(b) because although the measurement itself is repeatable, i.e., if we remeasure this device we get the same result, after several months work we are unable to produce another device showing the same behavior. The loss of the current plateaus in Fig. 4(b) bears further discussion. Comparing with Fig. 4(a) and earlier data, Device G pinch-off occurs before the current plateau normally begins. The initial depletion is very strong compared to the other devices; by $V_{g}=$ $+0.25 \mathrm{~V}$ the current has dropped to zero as opposed to the $30-$ $50 \%$ found in other devices without the $V_{g}=0$ conductivity being significantly less than normal. The very similar $I_{d}$ at $V_{g}=0$ for Devices $\mathrm{F}$ and $\mathrm{G}$ shows that the radically different pinch-off voltages are not due to a correspondingly large

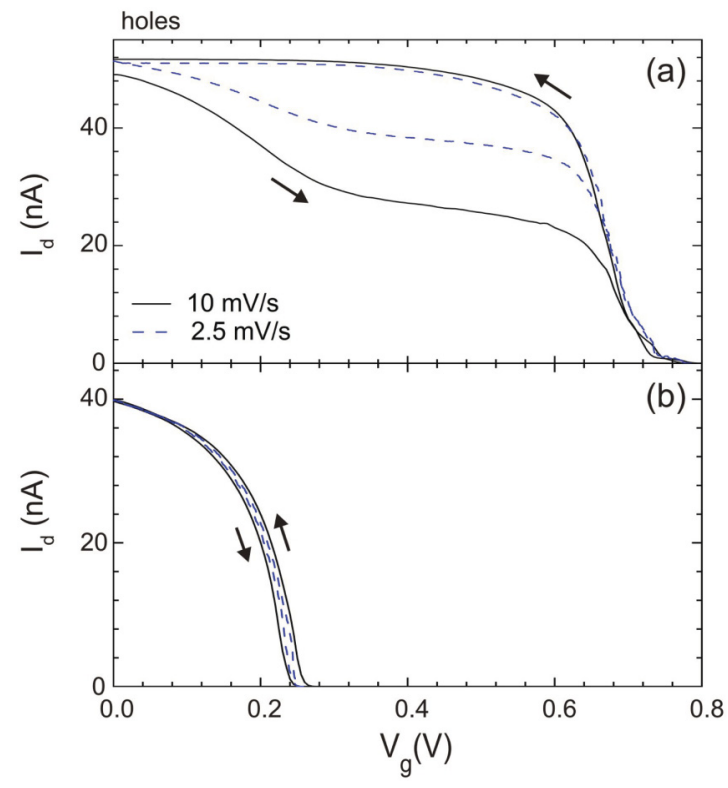

FIG. 4. (Color online) Channel current $I_{d}$ vs gate voltage $V_{g}$ for (a) Device F on heterostructure 1-h and (b) Device $G$ on heterostructure 1-h with sulfur passivation. The data in (a) are what we typically obtain with sulfur passivation, even with different treatments. ${ }^{35}$ The data in (b) are a repeatable measurement, but, to date, a nonreproducible device. We are still working to establish reliable conditions that produce this outcome. The solid black and dashed blue traces were obtained at sweep rates of 10 and $2.5 \mathrm{mV} / \mathrm{s}$. The arrows indicate hysteresis loop direction.

difference in $V_{g}=0$ carrier density. Although the rapid initial depletion in Device $G$ shows a strong reduction in the effect of the surface states on $I_{d}$ vs $V_{g}$, it is unclear this requires a major change in the surface-state spectrum. A reduction in surface-state density in the tail of the distribution causing the current plateau may suffice to ensure that depletion is completed before current plateau onset, giving the radical difference in pinch-off voltage between Devices F and G.

Putting Device $\mathrm{G}$ aside momentarily, our work suggests that passivation does little to reduce the hysteresis. ${ }^{35}$ An apparent initial explanation is that the passivation solution is ineffective on (311) surfaces-there are no prior studies on this GaAs surface, development has focused on the more commonly used (100), (110), and (111) surfaces. $^{34}$ We have performed comparative studies of the efficacy of sulfur passivation on (100) and (311) GaAs surfaces using photoluminescence measurements ${ }^{65}$ and find improvement in photoluminescence yield for both surfaces. ${ }^{35}$ Thus, while it is evident that passivation significantly affects the surface states, as expected from earlier work, ${ }^{32,33,61-65}$ this does not translate into a substantial change in the observed hysteresis. A possible explanation is that the single-dangling bonds present on the (311) surface interrupt the surface chemistry, reducing passivation treatment effectiveness. The importance of dangling-bond presentation to surface chemistry is well known; for example, it affects the incorporation probability of $\mathrm{Si}$ into $\mathrm{Al} / \mathrm{Ga}$ sites versus As sites during growth. ${ }^{66}$ XPS studies show that ammonium sulfide treatment of (100) GaAs surfaces leads to disulfide bridges between adjacent surface As atoms. ${ }^{62}$ It would be interesting to investigate whether this 
changes for the (311) surface; the corresponding effect on surface-state spectrum could be established using deep-level transient spectroscopy (DLTS). ${ }^{65}$ A focused surface chemistry study may ultimately reveal a passivation formulation that produces the improvement found for Device $G$ consistently.

Device $\mathrm{G}$ shows that passivation does not eliminate the hysteresis entirely and that surface states are not the whole story. There are additional data supporting this, for example, the addition of the $\mathrm{Al}_{2} \mathrm{O}_{3}$ layer to a (100)-oriented $n$-type heterostructure (Fig. 3) causes a threshold shift consistent with a large change in surface-state spectrum but does not introduce hysteresis, yet hysteresis is reported for (100)oriented $p$-type heterostructures ${ }^{27,28,31}$ with a loop direction (counterclockwise) consistent with our observations. These results suggest that dopants also play a role in the hysteresis; we now explore this possibility.

\section{EVIDENCE FOR THE ROLE OF DOPANTS}

The key to the remarkable stability and performance of $n$-type AlGaAs/GaAs heterostructures is the DX center, a deep trap consisting of a lattice-distorted $\mathrm{Si}_{\mathrm{Ga}}$ site. ${ }^{36}$ If an $n$-type heterostructure is warmed above $\sim 120 \mathrm{~K}$, the DX centers begin releasing their electrons, allowing the doping layer charge distribution to change. ${ }^{37,50,51}$ This provides an ideal system for studying whether charge motion in the doping layer generates hysteresis similar to that observed for $p$-type heterostructures. Gate hysteresis data from Device D at $T=120$ and $130 \mathrm{~K}$ are shown in Figs. 5(a) and 5(b). The $120 \mathrm{~K}$ data look like an intermediate between that in Figs. 3(a) and 3(b); the pinch-off voltage is slightly higher $(-0.3 \mathrm{~V}$ rather than $-0.2 \mathrm{~V})$ due to the increased temperature. While the apparent hysteresis in Fig. 3 runs counterclockwise, indicative of sweep-lag, the hysteresis in Fig. 5(a) runs clockwise, the direction corresponding to that observed in $p$-type heterostructures. The hysteresis becomes more pronounced at $130 \mathrm{~K}$ and its shape is interesting. As Fig. 5(c) illustrates, if one takes the characteristic shape obtained for holes, e.g., Fig. 1(b), removes the current plateaus (green dotted segments), closes the gap, and reflects about $V_{g}=0$ to account for carrier charge inversion, then a hysteresis loop with the same shape as that in Fig. 5(a) is obtained. The loss of the current plateaus is consistent with the lack of hysteresis in Figs. 3(a) and 3(b). This suggests the current plateaus are specific to holes, and likely a surface-state effect, consistent with Figs. 2 and 4(b). A notable feature of Fig. 5(a) is that the slow cycle (dashed blue trace) has a much higher pinch-off voltage than the fast cycle (solid black trace); this also occurs for $p$-type heterostructures at $T<1 \mathrm{~K}$, as discussed below.

Unfortunately, the gates in Device D begin to leak directly to the 2DEG for $T>130 \mathrm{~K}$, preventing higher temperature measurements. To go higher in $T$ and further explore this behavior, we performed the same study using Device E [Figs. 5(d)-5(g)]. Starting at $T=110 \mathrm{~K}$, no hysteresis appears; the data resemble those in Fig. 3(b) from Device D, albeit with a slightly higher pinch-off voltage. As $T$ is increased, very similar hysteresis to that in Device D emerges for $T>120 \mathrm{~K}$. The pinch-off voltage increases markedly with $T$, this limited the measurements to $T \leqslant 140 \mathrm{~K}$. Beyond this, the pinch-off voltage exceeds $\mathrm{Al}_{2} \mathrm{O}_{3}$ layer breakdown causing gate leakage. Device $\mathrm{E}$ gives similar
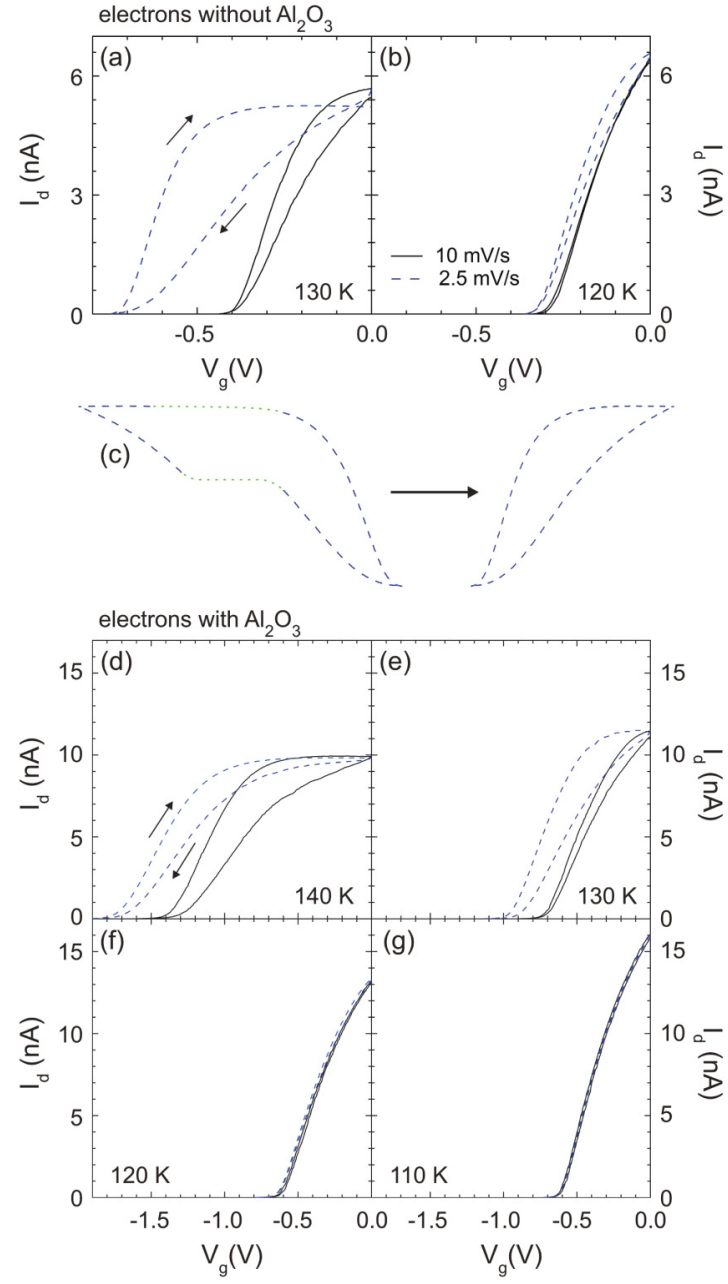

FIG. 5. (Color online) [(a) and (b)] Channel current $I_{d}$ vs gate voltage $V_{g}$ for Device $\mathrm{C}$ on heterostructure 1-e without $\mathrm{Al}_{2} \mathrm{O}_{3}$ at $T=$ (a) $130 \mathrm{~K}$ and (b) $120 \mathrm{~K}$. (c) A schematic illustrating an evident relationship between the hysteresis loop shape in hole (left) and electron devices (right), as discussed in the text. [(d)-(g)] $I$ vs $V_{g}$ for Device $\mathrm{D}$ on heterostructure 1-e with a $20 \mathrm{~nm} \mathrm{Al}{ }_{2} \mathrm{O}_{3}$ layer at $T=$ (d) $140 \mathrm{~K}$, (e) $130 \mathrm{~K}$, (f) $120 \mathrm{~K}$, and (g) $110 \mathrm{~K}$. In (a), (b), and (d)-(g) the solid black and dashed blue traces were obtained at sweep rates of 10 and $2.5 \mathrm{mV} / \mathrm{s}$. The arrows indicate hysteresis loop direction.

results to Device D, both in terms of the overall hysteresis loop shape and direction of travel and the trend for higher pinch-off voltage at lower sweep rate.

In Figs. 6(a)-6(h) we show the corresponding behavior for holes. As $T$ is reduced from $T=4.5 \mathrm{~K}$ to $T=260 \mathrm{mK}$, two obvious changes result: First, the current plateau shortens and drops to lower $I_{d}$. The plateau shortening and lowering are linked-if plateau onset shifts to higher $V_{g}$, then the low $V_{g}$ depletion proceeds further before the plateau appears. Second, for $T<1.14 \mathrm{~K}$, the pinch-off voltage for the slow trace significantly exceeds that for the fast trace. A careful inspection of Figs. 6(c)-6(e) reveals that this is caused by loss of the fast-trace current plateau, while the slow trace current plateau remains until $T<260 \mathrm{mK}$. For the fast-trace hysteresis loop at $T<1.14 \mathrm{~K}$, the hysteresis remains despite the loss of the current plateau and strongly resembles that in Figs. 4(b) and 5. This behavior clearly indicates that two processes are involved 

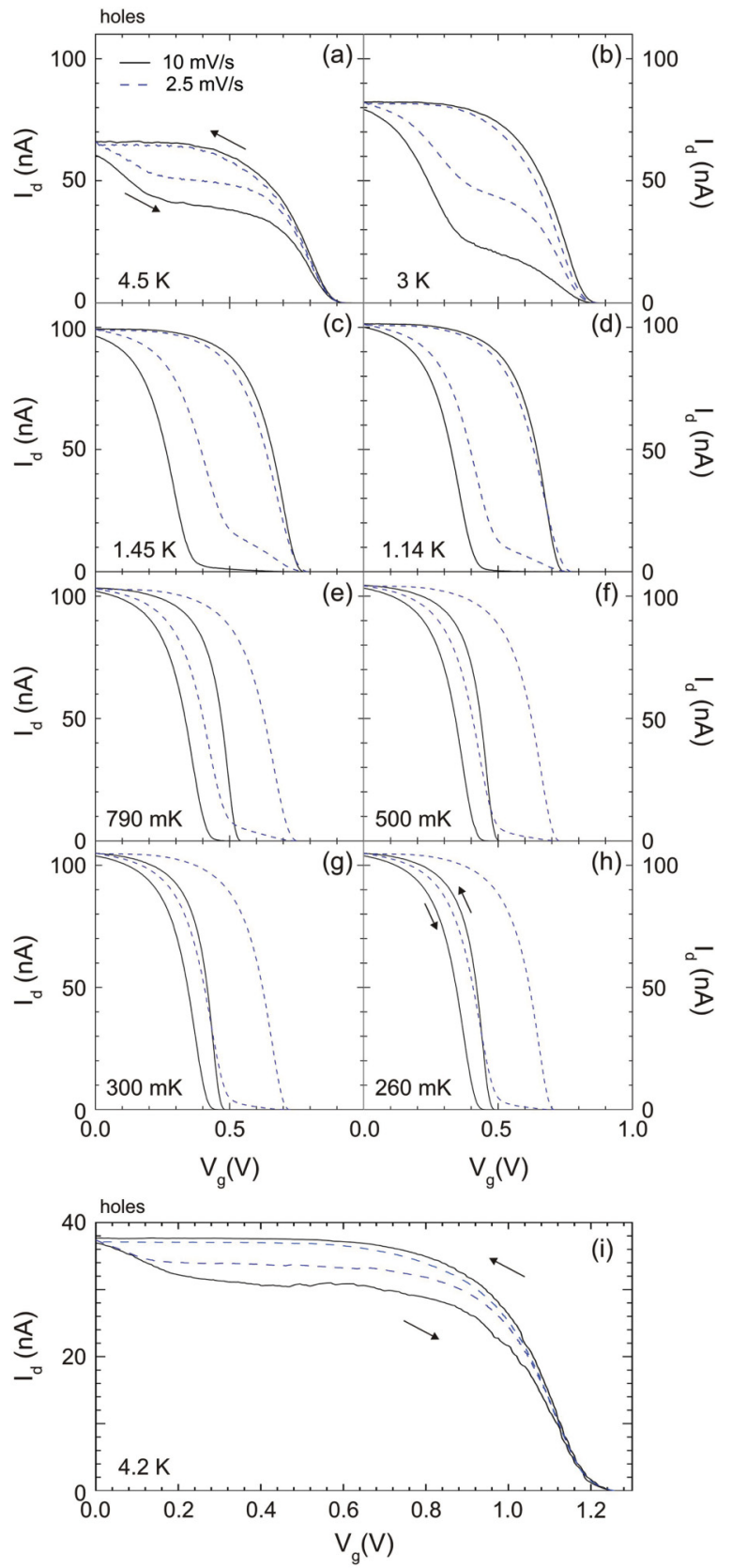

FIG. 6. (Color online) Channel current $I_{d}$ vs gate voltage $V_{g}$ for Device $\mathrm{F}$ on heterostructure 2-h without $\mathrm{Al}_{2} \mathrm{O}_{3}$ at $T=$ (a) $4.5 \mathrm{~K}$ and (b) $3.0 \mathrm{~K}, \quad$ (c) $1.45 \mathrm{~K}, \quad$ (d) $1.14 \mathrm{~K}$, (e) $790 \mathrm{mK}$, (f) $500 \mathrm{mK}$, (g) $300 \mathrm{mK}$, and (h) $260 \mathrm{mK}$. (i) $I_{d}$ vs $V_{g}$ for Device $\mathrm{H}$ on the $\delta$-doped heterostructure 3-h without $\mathrm{Al}_{2} \mathrm{O}_{3} /$ passivation at $T=4 \mathrm{~K}$. The solid black and dashed blue traces were obtained at sweep rates of 10 and $2.5 \mathrm{mV} / \mathrm{s}$. The arrows indicate hysteresis loop direction.

in generating the hysteresis - surface-state trapping and charge redistribution among the dopants, each with different time and energy scales. Attributing the current plateau to surface state trapping, it appears that the surface states "freeze-out" at $T<1 \mathrm{~K}$ leaving the dopant effects behind. This might explain the lack of hysteresis in the $\mathrm{HfO}_{2}$-insulated hole QPC studied by Csontos et al. ${ }^{31}$ compared to our $\mathrm{Al}_{2} \mathrm{O}_{3}$ device (Fig. 2). The data shown in Fig. 3(a) of Ref. 31 were obtained at $T=100 \mathrm{mK}$; here the surface-state component of the hysteresis is likely quenched, leaving only the hysteresis due to charge migration in the doping layer. This is why the hysteresis occurs primarily closer to pinch-off, consistent with Fig. 4(b), where surface-state hysteresis also appears to be quenched. The hysteresis loop direction in Ref. 31 is consistent with this explanation; data at higher $T$ for this device would be enlightening. To further demonstrate the influence of dopants in the hysteresis we discuss one final device. In Fig. 6(i) we present hysteresis data from Device $\mathrm{H}$, made on the $\delta$-doped heterostructure 3-h with Schottky gates on an unpassivated surface. The key difference is that the dopants have only a vertical spread of $<5 \mathrm{~nm}$ in Device $\mathrm{H}$, compared to $\sim 80 \mathrm{~nm}$ for Devices A-G. The $2 \mathrm{DHG}$ is closer to the surface $(47 \mathrm{~nm}$ for 3-h vs $120 \mathrm{~nm}$ for $1-\mathrm{h}$ ) and the $5 \mathrm{~nm}$ GaAs cap in 3-h is Si doped unlike heterostructures 1 and 2. Doping of the cap is normally performed to improve ohmic contact formation; however, the doping level is necessarily limited to prevent shorting of gates and ohmic contacts. Comparing Fig. 6(i) with Figs. 5(a) and 5(b), there are two notable differences. First and foremost, the hysteresis loop's vertical extent is reduced from $42 \%$ to $17 \%$. This reduction is likely due to the $>16$-fold reduction in dopant layer spread for Device $H$. Second, the pinch-off voltage and width of the current plateau are increased. A cap doping of $\sim 1-2 \times 10^{18} \mathrm{~cm}^{-3}$ corresponds to replacing only roughly 1 in every 220000 surface atoms with $\mathrm{Si}$. Considered alongside the sulfur passivation results, the cap doping is unlikely to be the dominant cause for the increase in current plateau width. It is more likely that the shallower $2 \mathrm{DHG}$ in 3-h exacerbates the surface-state contribution to the hysteresis. This would be consistent with scattering studies in shallow undoped $n$-type heterostructures where the surface-charge scattering contribution increased with reduced 2 DEG depth. ${ }^{38}$

\section{CONCLUSIONS}

We set out to identify the exact origin of the gate hysteresis in $p$-type AlGaAs/GaAs heterostructures-several divergent explanations exist in the literature and a better understanding will enable development of low-dimensional hole devices with improved stability/performance. A commonly accepted explanation is that gates on $p$-type heterostructures are inherently leaky. We show that this not the case; Schottky gates on $p$-type heterostructures are not significantly more leaky than Schottky gates on $n$-type heterostructures under reverse bias conditions. Hysteresis due to gate leakage in $p$-type heterostructures should give a clockwise hysteresis loop and the hysteresis we observe runs counterclockwise. We also find that the hysteresis becomes drastically worse rather than much better if the gates are insulated. We note that hysteresis was also still observed in QPCs with $\mathrm{HfO}_{2}$-insulated gates on C-doped $p$-type heterostructures, ${ }^{31}$ despite these producing improved tunability compared to equivalent Schottky-gated devices. The direction of the hysteresis loop in Ref. 31 is counterclockwise and is inconsistent with gate leakage. The measurements by Csontos et al. ${ }^{31}$ were obtained at very low temperature $T \sim 100 \mathrm{mK}$ and based on our data, we propose that this hysteresis may be due to charge redistribution in the dopant layer. 
Our work focused on the investigation of devices with insulated/uninsulated gates on custom-grown, matched electron and hole heterostructures. This relies on $\mathrm{Si}$ being an $n$-type dopant on (100) substrates and a $p$-type dopant on (311)A substrates. ${ }^{40}$ Despite the strong hysteresis in $p$-type devices at $T=4 \mathrm{~K}$, we observe no hysteresis in $n$-type devices until the temperature exceeds $T=120 \mathrm{~K}$, where the Si DX centers in the $n$-type heterostructure begin to detrap and migration of charge within the doping layer occurs. ${ }^{37,50-52}$ The hysteresis that emerges at $T>120 \mathrm{~K}$ for electrons bears a strong resemblance to that in holes at lower temperatures, particularly at $T<1 \mathrm{~K}$, where the current plateau at intermediate $V_{g}$ drops to $I_{d} \sim 0$ and is quenched. This correspondence, and the lack of hysteresis for electrons at $T \sim 4 \mathrm{~K}$, strongly suggests that surface states are not the sole cause of the hysteresis; migration of charge in the dopant layer is likely involved as well. We return to surface states following a discussion of dopants.

Comparatively little is known about the physics of $\mathrm{Si}$ acceptors in (311)A heterostructures. Although Si clearly acts as a substitutional acceptor, the presence and properties of acceptor complexes such as $\mathrm{Si}-\mathrm{X}$ is debated. ${ }^{53-59}$ Our data cannot provide insight at the atomic level, but there are clearly no acceptor sites in a $p$-type heterostructure's doping layer that act like the deep-trapping DX centers in $n$-type heterostructures. Regarding Si-X specifically, we can draw two conclusions: If $\mathrm{Si}-\mathrm{X}$ exists in $\mathrm{Al}_{0.33} \mathrm{Ga}_{0.67} \mathrm{As}$ in (311)A heterostructures, then it must be a very shallow trap if present at high density (more than 100 times shallower than DX), and only a deep trap if it is present at such low density that it cannot "freeze" the doping layer's charge configuration. While the lack of DX-like deep traps answers the obvious question of why $p$-type heterostructures are so unstable/hysteretic under gating, it is interesting to invert the thinking and consider instead why $n$-type heterostructures are often so impressively stable. The answer is clear-the deep-trapping DX centers "lock down" the vast majority of free charge in the dopant layer-and inspirational: One way to stabilize modulationdoped $p$-type heterostructures may be to deliberately poison the modulation doping layer with deep-trapping sites, perhaps by engineering the growth conditions to obtain a high density of deep-trapping $\mathrm{Si}$ acceptor complexes or, failing that, by codoping with transition metal impurities, e.g., $\mathrm{Cu}, \mathrm{Fe}, \mathrm{Ni}$, or $\mathrm{Zn} .{ }^{67-69} \mathrm{Cu}$ is probably more optimal than $\mathrm{Fe}$ or $\mathrm{Ni}$, which may bring magnetic side effects, and $\mathrm{Zn}$, which is a rapid diffuser in GaAs and may be incompatible with ohmic contact formation.

The changes in hysteresis obtained by changing insulator composition or surface passivation are small compared to the differences arising between the presence/absence of a gate insulator. One possible explanation is that the metal/GaAs interface quenches the surface-state density, either chemically by forming Ti-O-Ga or Ti-O-As bonds (or Ti-S-Ga or Ti-S-As bonds) or physically by providing a nearby high electron density that partially screens the surface states. ${ }^{70,71}$ This is in the same spirit as the addition of a doping layer to compensate the surface states in ultrahigh mobility $n$-type AlGaAs/GaAs heterostructures. ${ }^{72,73}$ This idea could be applied here with the lightly doped cap in 3-h replaced by adding a uniformly doped layer between the cap and modulation doping for surface-state compensation.
Another alternative is to abandon Schottky gates and use a degenerately doped cap as a semiconductor gate. ${ }^{74-77}$ This effectively places the gate underneath the semiconductor surface, enabling the gate to screen the $2 \mathrm{DHG}$ from the surface states. Indeed, this explains the high stability and lack of hysteresis in undoped $p$-type semiconductor-insulatorsemiconductor field-effect transistor (SISFET) devices. ${ }^{17,77-80}$ There both hysteresis contributions are dealt with-the modulation doping is removed and the gate screens the surface states. Given the success of undoped SISFETs, one might ask: Why bother making semiconductor-gated modulation-doped devices? In undoped SISFETs it is essential that the gate overlaps the ohmic contacts; this makes fabrication more difficult and lowers yield. ${ }^{76}$ This overlap is unnecessary in modulation-doped devices. Hence, if the doping layer charge migration issue described earlier can be successfully overcome, modulation-doped semiconductor-gated structures may provide a formidable platform for studying low-dimensional hole devices.

\section{ACKNOWLEDGMENTS}

This work was funded by Australian Research Council Grants No. DP0772946, No. DP0877208, No. FT0990285, and No. FT110100072. D.R. and A.D.W. acknowledge support from DFT SPP1285 and BMBF QuaHL-Rep 01BQ1035. This work was performed in part using the NSW and ACT nodes of the Australian National Fabrication Facility (ANFF). We thank L. Eaves for helpful discussions on DX centers and L. H. Ho for the initial measurements that motivated our interest in this problem. D.W. and D.C. contributed equally to the work.

\section{APPENDIX A: DETAILED MATERIALS AND METHODS}

Experiments were performed on five separate Al$\mathrm{GaAs} / \mathrm{GaAs}$ heterostructures grown by molecular beam epitaxy, denoted 1-e, 1-h, 2-e, 2-h, and 3-h. The first four were produced in two separate growth runs, each performed onto side-by-side halves of 2-inch-diameter semi-insulating GaAs substrate, one (100) oriented and the other (311) oriented, to give "matched" electron (-e) and hole (-h) heterostructures, respectively. Production of electron and hole heterostructures in a single growth is enabled by the facet-specific amphoteric nature of Si dopants in AlGaAs [ $n$-type on (100) and $p$-type on (311)]. ${ }^{40}$ Both growth runs have a nominally identical epilayer structure, the active region consisting of $650 \mathrm{~nm}$ undoped GaAs, $35 \mathrm{~nm}$ undoped $\mathrm{Al}_{0.34} \mathrm{Ga}_{0.66} \mathrm{As}$, $80 \mathrm{~nm} \mathrm{Si}$ doped $\mathrm{Al}_{0.34} \mathrm{Ga}_{0.66} \mathrm{As}$, and a $5 \mathrm{~nm}$ undoped $\mathrm{GaAs}$ cap. The $\delta$-doped wafer 3-h begins with $689 \mathrm{~nm}$ undoped GaAs and $21 \mathrm{~nm}$ undoped $\mathrm{Al}_{0.33} \mathrm{Ga}_{0.67} \mathrm{As}$ grown at $690^{\circ} \mathrm{C}$. Growth is then interrupted and the substrate cooled to $580^{\circ} \mathrm{C}$. The $\mathrm{Si}$ source is opened for $180 \mathrm{~s}$ and then $5 \mathrm{~nm}$ of undoped $\mathrm{Al}_{0.33} \mathrm{Ga}_{0.67} \mathrm{As}$ is grown. Growth is interrupted again to return the substrate to $690^{\circ} \mathrm{C}$ before finishing the device with $16 \mathrm{~nm}$ of undoped $\mathrm{Al}_{0.33} \mathrm{Ga}_{0.67} \mathrm{As}$ and a $5 \mathrm{~nm}$ GaAs cap. The devices have typical carrier densities $\sim 1.3 \times 10^{11} \mathrm{~cm}^{-2}$ and mobilities $500000 \mathrm{~cm}^{2} / \mathrm{Vs}$ at temperature $T=4 \mathrm{~K}$; see Table I for specific values for each heterostructure at $T=300 \mathrm{mK}$ using four-terminal Shubnikov-de Haas and Hall resistivity measurements. All measurements were performed 
TABLE I. The Bochum wafer number, electron/hole density, and mobility for the five AlGaAs/GaAs heterostructures studied at $T \sim 300 \mathrm{mK}$.

\begin{tabular}{lcccr}
\hline \hline Heterostructure & $\begin{array}{c}\text { Bochum wafer } \\
\text { number }\end{array}$ & Carrier & $\begin{array}{c}\text { Density } \\
\left(\mathrm{cm}^{-2}\right)\end{array}$ & $\begin{array}{r}\text { Mobility } \\
\left(\mathrm{cm}^{2} / \mathrm{Vs}\right)\end{array}$ \\
\hline $1-\mathrm{e}$ & $13473-\mathrm{e}$ & Electrons & $1.20 \times 10^{11}$ & 640000 \\
$1-\mathrm{h}$ & $13473-\mathrm{h}$ & Holes & $1.94 \times 10^{11}$ & 805800 \\
$2-\mathrm{e}$ & $13516-\mathrm{e}$ & Electrons & $1.46 \times 10^{11}$ & 345000 \\
$2-\mathrm{h}$ & $13516-\mathrm{h}$ & Holes & $1.64 \times 10^{11}$ & 1030000 \\
3-h & $13483-\mathrm{h}$ & Holes & $1.74 \times 10^{11}$ & 1250000 \\
\hline \hline
\end{tabular}

in the dark. Details for each of the eight devices studied are presented in Table II.

Hall bars with a $140 \mathrm{~nm}$-high mesa were produced using photolithography and a 2:1:20 buffered $\mathrm{HF}: \mathrm{H}_{2} \mathrm{O}_{2}: \mathrm{H}_{2} \mathrm{O}$ wet etch. The buffered HF solution is 7:1 $\mathrm{NH}_{4} \mathrm{~F}: \mathrm{HF}$. The photolithographically defined ohmic contacts consist of $150 \mathrm{~nm}$ AuBe alloy for $p$-type contacts and a stack containing $5 \mathrm{~nm}$ $\mathrm{Ni}, 35 \mathrm{~nm} \mathrm{Ge}, 72 \mathrm{~nm} \mathrm{Au}, 18 \mathrm{~nm} \mathrm{Ni}$, and $50 \mathrm{~nm}$ Au for $n$-type contacts. Contacts were annealed at $490^{\circ} \mathrm{C}$ for $90 \mathrm{~s}$ ( $p$-type) and $430^{\circ} \mathrm{C}$ for $30 \mathrm{~s}$ ( $n$-type). The photolithographically defined gates consist of $20 \mathrm{~nm} \mathrm{Ti}$ and $100 \mathrm{~nm}$ Au deposited after the ohmic contact anneal. Figure 7(a) shows an optical micrograph of a completed device.

$\mathrm{Al}_{2} \mathrm{O}_{3}$-insulated gate samples were produced by adding the following steps between mesa etch and ohmic contact metallization. A $20 \mathrm{~nm} \mathrm{Al}_{2} \mathrm{O}_{3}$ layer was deposited using $\left(\mathrm{CH}_{3}\right)_{3} \mathrm{Al}$ and $\mathrm{H}_{2} \mathrm{O}$ gaseous precursors at $200^{\circ} \mathrm{C}$ in a Cambridge Nanotech Savannah 100 Atomic Layer Deposition (ALD) system. Access to the heterostructure for the ohmic contacts was obtained by a $60 \mathrm{~s}$ buffered $\mathrm{HF}$ etch $\left(7: 1 \mathrm{NH}_{4} \mathrm{~F}: \mathrm{HF}\right.$ in $\left.\mathrm{H}_{2} \mathrm{O}\right)$ following photolithographic definition of the contact regions and prior to metallization. In the absence of $\mathrm{H}_{2} \mathrm{O}_{2}$ this etch self-terminates, stripping the native GaAs surface oxide in the process. The openings in the $\mathrm{Al}_{2} \mathrm{O}_{3}$ layer are visible optically [Fig. 7(b)] but are clearer using scanning electron microscopy [Fig. 7(c)]. Although traces of residual oxide remain, they do not adversely affect ohmic contact formation.

The polyimide-insulated gate sample was produced with an added step between the ohmic contact anneal and gate deposition. A $140 \mathrm{~nm}$ layer of patterned polyimide is obtained by spin-coating a diluted mixture (1:1.4) of photo-processable polyimide (HD Microsystems HD-4104) in thinner (HD Microsystems T-9039), performing a $65^{\circ} \mathrm{C}$ soft-bake for $90 \mathrm{~s}$, exposing/developing aligned ohmic contact openings,

TABLE II. Details for the eight devices studied. Mod = Modulation doped, $\delta=$ delta doped.

\begin{tabular}{lcccc}
\hline \hline Device & Heterostructure & Doping & Insulator & Passivation \\
\hline A & $1-h$ & Mod & No & No \\
B & $1-h$ & $M o d$ & $\mathrm{Al}_{2} \mathrm{O}_{3}$ & No \\
C & $2-h$ & $M o d$ & Polyimide & No \\
D & $1-\mathrm{e}$ & $\mathrm{Mod}$ & No & No \\
E & $1-\mathrm{e}$ & $\mathrm{Mod}$ & $\mathrm{Al}_{2} \mathrm{O}_{3}$ & No \\
F & $1-\mathrm{h}$ & $\mathrm{Mod}$ & No & Yes \\
$\mathrm{G}$ & $1-\mathrm{h}$ & $\mathrm{Mod}$ & No & Yes \\
$\mathrm{H}$ & $3-\mathrm{h}$ & $\delta$ & No & No \\
\hline \hline
\end{tabular}
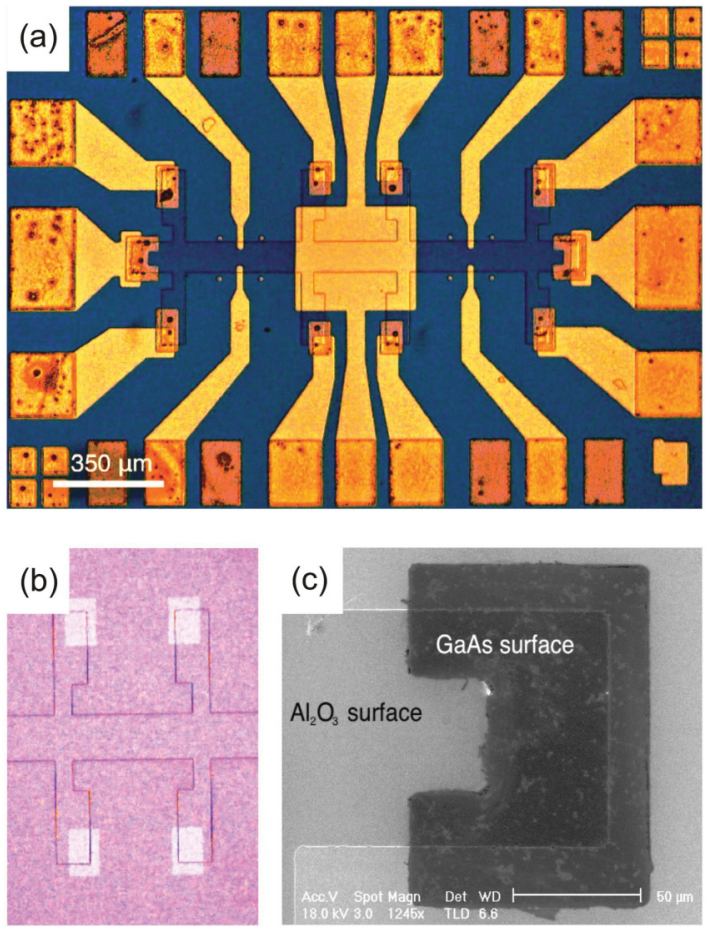

FIG. 7. (Color online) (a) Optical micrograph of a completed device with 10 ohmic contacts ( 3 at each end and 4 near the middle) and a single top-gate covering the midsection of the Hall bar with interconnects at top and bottom. The remaining four metal gates were not used. (b) Nomarski phase-contrast optical micrograph showing four etched penetrations (off-white) in the $20 \mathrm{~nm} \mathrm{Al} \mathrm{O}_{3}$ layer (pink) obtained using a buffered HF etch. These correspond to the four central ohmic contacts in (a). (c) Scanning electron micrograph of the etch penetration (dark) in the $\mathrm{Al}_{2} \mathrm{O}_{3}$ layer (light) for the ohmic contact at the far right of the Hall bar in (a). (b) and (c) were obtained after the buffered HF etch and before ohmic metallization.

and finishing with a $250^{\circ} \mathrm{C}$ hard-bake for 60 min under $1 \mathrm{~atm} \mathrm{~N}_{2}$.

The sulfur passivation treatment was performed immediately before gate deposition. A stock solution of $\left(\mathrm{NH}_{4}\right)_{2} \mathrm{~S}_{x}$ was prepared by adding $9.62 \mathrm{~g}$ of elemental sulfur (Aldrich) to $100 \mathrm{~mL}$ of $20 \%\left(\mathrm{NH}_{4}\right)_{2} \mathrm{~S}$ solution (Aldrich) and mixing until completely dissolved. The passivation solution was a $0.5 \%$ dilution of the stock solution in deionized water (Millipore). Passivation was performed by immersion for $2 \min$ in $3-5 \mathrm{~mL}$ of passivation solution heated to $40^{\circ} \mathrm{C}$ in a water bath, followed by a deionized water rinse. The sample is stored under deionized water during transfer to the vacuum evaporator. The total time between passivation and sample at vacuum was $<30 \mathrm{~min}$, with the sample exposed to air for no longer than a few minutes during evaporator loading and pump-down.

Electrical measurements at $T \geqslant 4 \mathrm{~K}$ were obtained using a liquid helium dip station, with $T>4 \mathrm{~K}$ achieved using the natural stratification of the He atmosphere inside the dewar. Data at $0.25-4 \mathrm{~K}$ were obtained using an Oxford Instruments Heliox ${ }^{3} \mathrm{He}$ cryostat. Standard two- and four-terminal ac lock-in techniques were used to measure the conducting channel's drain current $I_{d}$, typically with a $100 \mu \mathrm{V}$ constant voltage excitation at $73.3 \mathrm{~Hz}$ applied to the source. Pinch-off (i.e., $I_{d}=0$ ) is interpreted as $I_{d}<10 \mathrm{pA}$; this triggers the 


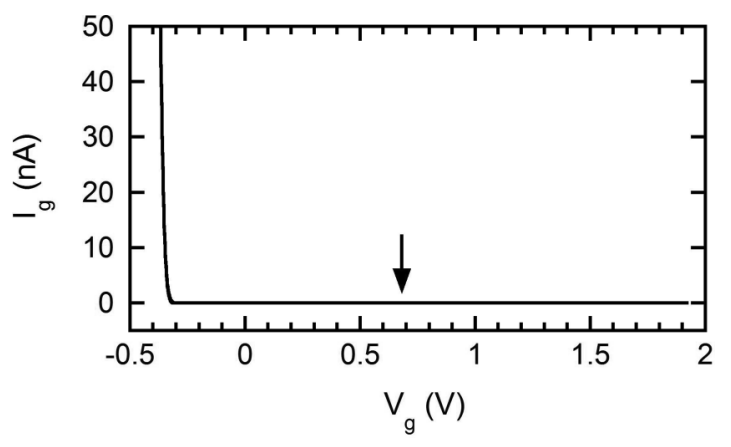

FIG. 8. Gate leakage current $I_{g}$ on a linear axis vs gate bias $V_{g}$ for a Schottky-gated modulation-doped $p$-type heterostructure. The data match those shown in Fig. 1(a). At positive $V_{g}, I_{g}$ remains less than $50 \mathrm{pA}$ to $V_{g}=+2 \mathrm{~V}$, sufficient to achieve pinch-off for all uninsulated gate devices studied. In an $n$-type heterostructure, gate leakage would normally occur at $V_{g}=+0.68 \mathrm{~V}$ (indicated by the arrow) and is suppressed for negative $V_{g}$.

software to commence the downsweep to avoid pointlessly driving the device beyond pinch-off. The gate bias $V_{g}$ was applied using a Keithley 2400 source-measure unit enabling continuous measurement of gate leakage current $I_{g}$ down to $100 \mathrm{pA}$. This instrument has a built-in current limiter, with $V_{g}$ curtailed to keep $I_{g}$ at a specified limit even if a higher $V_{g}$ if requested.

\section{APPENDIX B: LEAKAGE CHARACTERISTICS OF SCHOTTKY GATES ON $p$-TYPE HETEROSTRUCTURES}

Figure 8 shows the data in Fig. 1(a) plotted on a linear-linear scale for comparison. The gate leakage current $I_{g}$ is $<50 \mathrm{pA}$ for the entire range $0<V_{g}<+2 \mathrm{~V}$.

\section{APPENDIX C: ANALYSIS OF THE HYSTERESIS IN FIG. 1(b)}

Figure 9 shows the vertical extent of the hysteresis loop and the slope of the upsweeps vs $V_{g}$. The slope curves for the down sweeps are very similar to those in Fig. 9 except there is less structure at low $V_{g}$ and the peak at higher $V_{g}$ is sharper and shifted to more positive $V_{g}$. If the hysteresis is caused by sweep lag, one would expect the maximum vertical extent in the hysteresis to coincide in $V_{g}$ with the maximum slope $d I / d V_{g}$. This is clearly not the case, supporting our assessment that the hysteresis is not caused by sweep lag.

\section{APPENDIX D: REPRODUCIBILITY OF THE HYSTERESIS RESULT FROM DEVICE B-POLYIMIDE-INSULATED GATE ON A $p$-TYPE HETEROSTRUCTURE}

Figure 10 shows hysteresis loop data for Device B2, which is nominally identical to Device B aside from a change in the gate design used. The gate in Device B2 covers less area. In both cases, the gate does not extend to the edges of the $\mathrm{Al}_{2} \mathrm{O}_{3}$ layer; hence, in neither case is leakage by proximal direct shorting between gate and ohmic contact. The characteristics are very similar to those in Device B, and the breakdown volt-
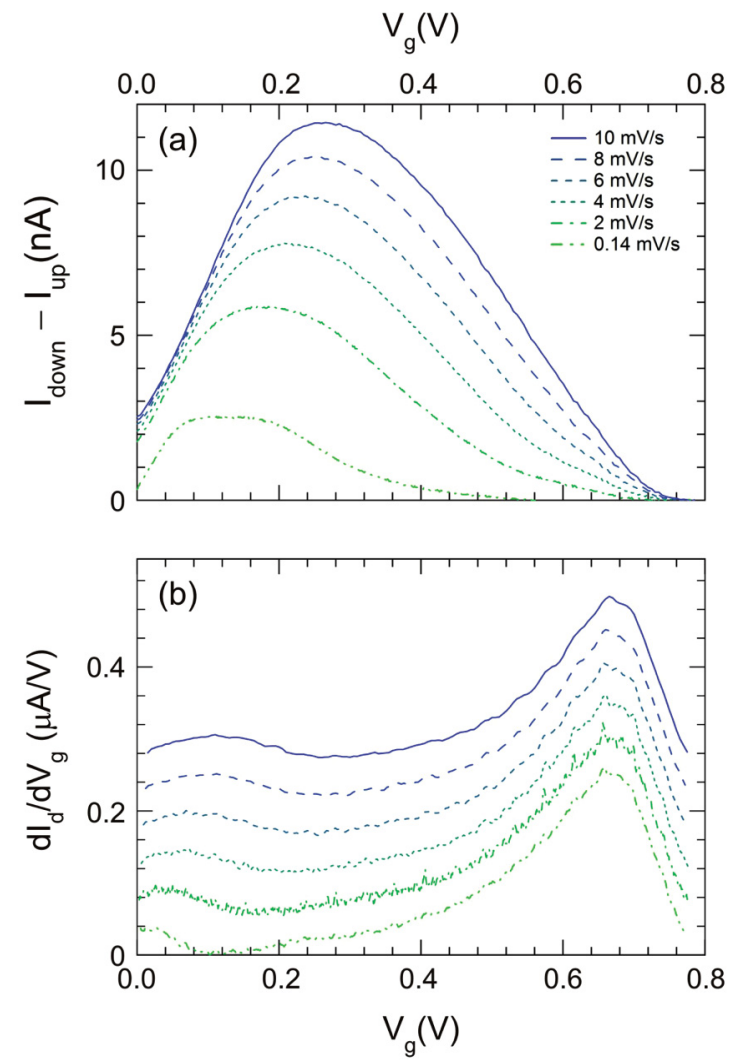

FIG. 9. (Color online) (a) The hysteresis loop vertical extent $I_{\text {down }}-I_{\text {up }}$ and (b) the numerical transconductance $d I_{d} / d V_{g}$ for the upsweeps vs gate voltage $V_{g}$. The traces in (b) are sequentially offset vertically by +0.05 with increasing sweep rate for clarity.

age is of a similar magnitude, $\sim 5 \mathrm{~V}$. Repeated sweeps appear to increase the breakdown voltage, enabling the device to slowly progress towards pinch-off. The exact cause for this is unclear.

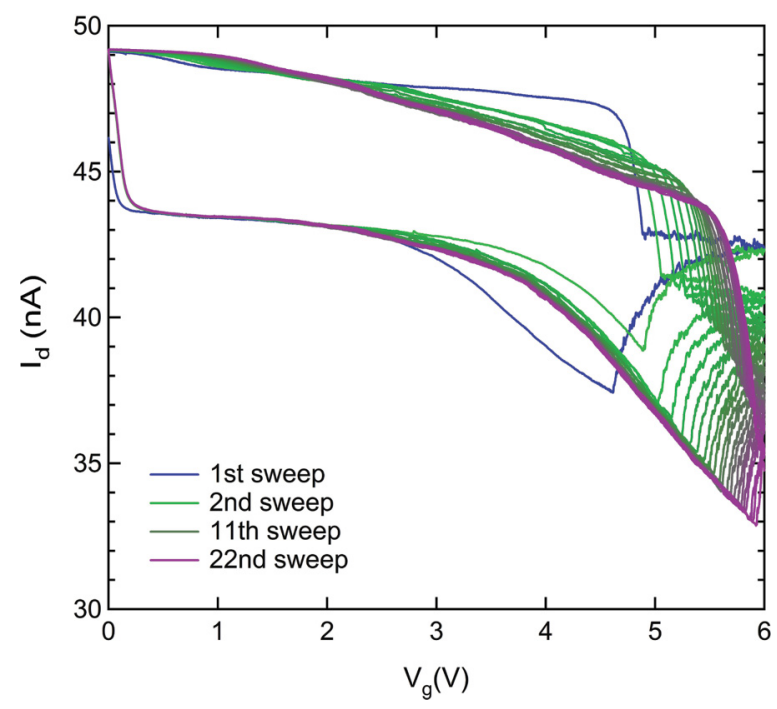

FIG. 10. (Color online) Channel current $I_{d}$ vs gate voltage $V_{g}$ for 22 hysteresis loop sweeps of Device B2. The first sweep is show in blue, and the second to twenty-second sweeps evolve continuously in color from green to magenta. The sweep rate in each case is $5 \mathrm{mV} / \mathrm{s}$. The points of discontinuity at high $V_{g}$ are where gate voltage current limiting comes on/off for the up/downsweep. 
The radical effect that the composition and properties of the insulator-semiconductor interface can have on transistor performance is well known for organic FETs. ${ }^{49}$ Due to high interface-state densities, inorganic oxide insulators are particularly troublesome, causing significant hysteresis and shifts in threshold voltage. Polymeric insulators such as polyimide often bring significant improvement. To explore whether the current plateau and difficulty in attaining pinch-off in Device B is tied to insulator composition, we studied Device $\mathrm{C}$ containing gates insulated with a $140 \mathrm{~nm}$-thick polyimide layer on 2-h. Figures 2(b) and 11(a) show $I_{d}$ and $I_{g}$ vs $V_{g}$ for this device. Pinch-off cannot be achieved in Device C, largely due to the much lower breakdown field for the polyimide insulator. Breakdown occurs for $V_{g} \gtrsim+2.8 \mathrm{~V}$, and once $I_{g}$ reaches $50 \mathrm{nA}$ at $V_{g} \sim+3.4 \mathrm{~V}$, the voltage source holds the gate bias fixed, as in Fig. 2. Thus, the data at $V_{g} \gtrsim 3.4 \mathrm{~V}$ in Fig. 11(a) should instead be considered as $I_{d}$ vs time $t$ at constant $V_{g}$. Here each minor subtick corresponds to a time of $10 \mathrm{~s}$ for the $10 \mathrm{mV} / \mathrm{s}$ trace and $20 \mathrm{~s}$ for the $5 \mathrm{mV} / \mathrm{s}$ trace. We show the data obtained beyond pinch-off in Fig. 11 to better facilitate comparison with Fig. 2 and to highlight the equilibration behavior that occurs when a sweep is stopped. The $I_{d}$ vs $V_{g}$ characteristics for Device $\mathrm{C}$ bear a striking resemblance to those of Device B (Figs. 2 and 10). On the upsweep there is an initial drop in current that plateaus for intermediate $V_{g}$, followed by a recovery in $I_{d}$ when $V_{g}$ is held constant. This is consistent with slow accumulation of net negative charge between the gate and $2 \mathrm{DHG}$, as per the explanation for hysteresis loop shape/direction in Sec. III D. The downsweep gives an initial rapid rise in $I_{d}$ that plateaus as $V_{g}$ approaches zero again, and as in Fig. 2, there is a distinct asymmetry between the upsweeps and downsweeps regarding the dependence of the path taken on sweep rate. Pinch-off

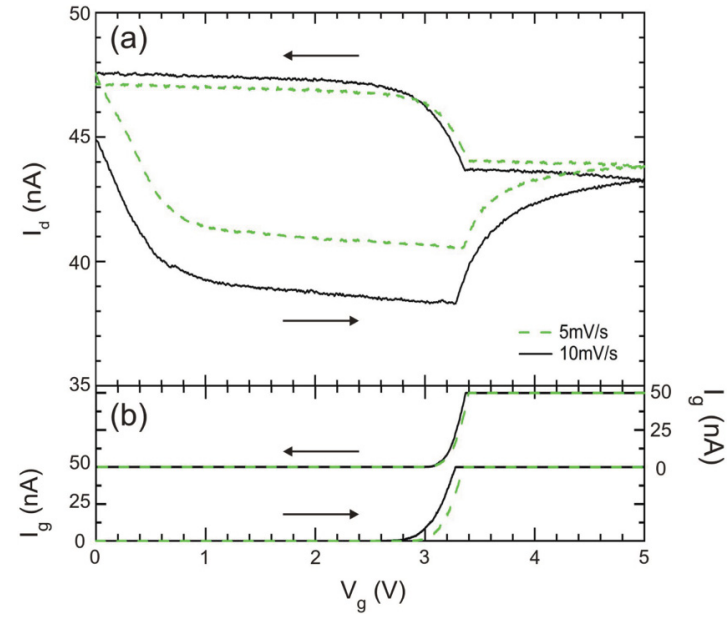

FIG. 11. (Color online) (a) Channel current $I_{d}$ and (b) gate leakage current $I_{g}$ vs gate voltage $V_{g}$ at two different gate sweep rates for a Device $\mathrm{C}$ featuring a $140 \mathrm{~nm}$ polyimide layer on $2-\mathrm{h}$. The horizontal arrows indicate the direction of travel around the hysteresis loop. In (b) the left/right axis and lower/upper set of data are for the sweep to/from positive $V_{g}$, respectively. Note that once $I_{g}$ reaches $50 \mathrm{nA}$ the gate voltage source implements current limiting by holding $V_{g}$ fixed. Hence, for $V_{g} \gtrsim+3.2 \mathrm{~V}$ the data in (a) should be considered as $I_{d}$ vs time $t$ at fixed $V_{g}$. To aid in converting the data at $V_{g} \gtrsim+3.2 \mathrm{~V}$ into time, each $0.2 \mathrm{~V}$ minor tick in the figure corresponds to 40 and $20 \mathrm{~s}$ for sweep rates of 5 and $10 \mathrm{mV} / \mathrm{s}$, respectively.

occurs beyond $V_{g} \sim+3.4 \mathrm{~V}$, thus, the shift in pinch-off bias must exceed $\sim 3.4 / 0.77=4.41$. The capacitive conversion factor for this device $V_{g}^{\mathrm{Ins}}=5.18 V_{g}$ assuming $140 \mathrm{~nm}$ of polyimide with dielectric constant 3.36 , giving an anticipated pinch-off voltage of $\sim+4.15 \mathrm{~V}$. *adam.micolich@nanoelectronics.physics.unsw.edu.au

${ }^{1}$ F. Capasso and S. Datta, Phys. Today 43, 74 (1990).

${ }^{2}$ S. M. Goodnick and J. P. Bird, IEEE Trans. Nanotech. 2, 368 (2003).

${ }^{3}$ T. Ando, A. B. Fowler, and F. Stern, Rev. Mod. Phys. 54, 437 (1982).

${ }^{4}$ H. L. Störmer, Rev. Mod. Phys. 71, 875 (1999).

${ }^{5}$ C. W. J. Beenakker and H. van Houten, Solid State Phys. 44, 1 (1991).

${ }^{6}$ A. P. Micolich, J. Phys.: Condens. Matter 23, 443201 (2011).

${ }^{7}$ L. P. Kouwenhoven, D. G. Austing, and S. Tarucha, Rep. Prog. Phys. 64, 701 (2001).

${ }^{8}$ W. G. van der Wiel, S. De Franceschi, J. M. Elzerman, T. Fujisawa, S. Tarucha, and L. P. Kouwenhoven, Rev. Mod. Phys. 75, 1 (2003).

${ }^{9}$ R. Hanson, L. P. Kouwenhoven, J. R. Petta, S. Tarucha, and L. M. K. Vandersypen, Rev. Mod. Phys. 79, 1217 (2007).

${ }^{10}$ Y. Hanein, U. Meirav, D. Shahar, C. C. Li, D. C. Tsui, and H. Shtrikman, Phys. Rev. Lett. 80, 1288 (1998).

${ }^{11}$ S. J. Papadakis, E. P. De Poortere, H. C. Manoharan, M. Shayegan, and R. Winkler, Science 283, 2056 (1999).

${ }^{12}$ E. Tutuc, M. Shayegan, and D. A. Huse, Phys. Rev. Lett. 93, 036802 (2004).

${ }^{13}$ W. R. Clarke, A. P. Micolich, A. R. Hamilton, M. Y. Simmons, C. B. Hanna, J. R. Rodriguez, M. Pepper, and D. A. Ritchie, Phys. Rev. B 71, 081304 (2005).
${ }^{14}$ R. Winkler, S. J. Papadakis, E. P. De Poortere, and M. Shayegan, Phys. Rev. Lett. 85, 4574 (2000).

${ }^{15}$ R. Winkler, E. Tutuc, S. J. Papadakis, S. Melinte, M. Shayegan, D. Wasserman, and S. A. Lyon, Phys. Rev. B 72, 195321 (2005).

${ }^{16}$ R. Danneau, O. Klochan, W. R. Clarke, L. H. Ho, A. P. Micolich, M. Y. Simmons, A. R. Hamilton, M. Pepper, and D. A. Ritchie, Phys. Rev. Lett. 100, 016403 (2008).

${ }^{17}$ O. Klochan, A. P. Micolich, A. R. Hamilton, K. Trunov, D. Reuter, and A. D. Wieck, Phys. Rev. Lett. 107, 076805 (2011).

${ }^{18}$ J.-B. Yau, E. P. De Poortere, and M. Shayegan, Phys. Rev. Lett. 88, 146801 (2002).

${ }^{19}$ Z. K. Keane, M. C. Godfrey, J. C. H. Chen, S. Fricke, O. Klochan, A. M. Burke, A. P. Micolich, H. E. Beere, D. A. Ritchie, K. V. Trunov, D. Reuter, A. D. Wieck, and A. R. Hamilton, Nano Lett. 11, 3147 (2011).

${ }^{20}$ D. Heiss, S. Schaeck, H. Huebl, M. Bichler, G. Abstreiter, J. J. Finley, D. V. Bulaev, and D. Loss, Phys. Rev. B 76, 241306 (2007).

${ }^{21}$ B. D. Gerardot, D. Brunner, P. A. Dalgarno, P. Öhberg, S. Seidl, M. Kroner, K. Karrai, N. G. Stolz, P. M. Petroff, and R. J. Warburton, Nature 451, 441 (2008).

${ }^{22}$ D. V. Bulaev and D. Loss, Phys. Rev. Lett. 95, 076805 (2005).

${ }^{23}$ I. Zailer, J. E. F. Frost, C. J. B. Ford, M. Pepper, M. Y. Simmons, D. A. Ritchie, J. T. Nicholls, and G. A. C. Jones, Phys. Rev. B 49, 5101 (1994). 
${ }^{24}$ A. J. Daneshvar, C. J. B. Ford, A. R. Hamilton, M. Y. Simmons, M. Pepper, and D. A. Ritchie, Phys. Rev. B 55, 13409 (1997).

${ }^{25}$ L. P. Rokhinson, D. C. Tsui, L. N. Pfeiffer, and K. W. West, Superlatt. Microstruct. 32, 99 (2002).

${ }^{26}$ B. Grbić, R. Leturcq, K. Ensslin, D. Reuter, and A. D. Wieck, Appl. Phys. Lett. 97, 232108 (2005).

${ }^{27}$ B. Grbić, Ph.D. thesis, ETH Zurich, 2007.

${ }^{28}$ C. Gerl, J. Bauer, and W. Wegscheider, J. Cryst. Growth 301, 145 (2007).

${ }^{29}$ I. Zailer, Ph.D. thesis, University of Cambridge, 1994.

${ }^{30}$ A. J. Daneshvar, Ph.D. thesis, University of Cambridge, 1998.

${ }^{31}$ M. Csontos, Y. Komijani, I. Shorubalko, K. Ensslin, D. Reuter, and A. D. Wieck, Appl. Phys. Lett. 97, 022110 (2010).

${ }^{32}$ C. J. Sandroff, R. N. Nottenburg, J.-C. Bischoff, and R. Bhat, Appl. Phys. Lett. 51, 33 (1987).

${ }^{33}$ E. Yablonovitch, C. J. Sandroff, R. Bhat, and T. Gmitter, Appl. Phys. Lett. 51, 439 (1987).

${ }^{34}$ M. V. Lebedev, Prog. Surf. Sci. 70, 153 (2002).

${ }^{35}$ D. Carrad et al. (to be published).

${ }^{36}$ P. M. Mooney, J. Appl. Phys. 67, R1 (1990); P. M. Mooney, Semicond. Sci. Technol. 6, B1 (1991).

${ }^{37}$ B. C. Scannell, I. Pilgrim, A. M. See, R. D. Montgomery, P. K. Morse, M. S. Fairbanks, C. A. Marlow, H. Linke, I. Farrer, D. A. Ritchie, A. R. Hamilton, A. P. Micolich, L. Eaves, and R. P. Taylor, Phys. Rev. B 85, 195319 (2012).

${ }^{38}$ W. Y. Mak, K. Das Gupta, H. E. Beere, I. Farrer, F. Sfigakis, and D. A. Ritchie, Appl. Phys. Lett. 97, 242107 (2010).

${ }^{39}$ C. Buizert, F. H. L. Koppens, M. Pioro-Ladrière, H.-P. Tranitz, I. T. Vink, S. Tarucha, W. Wegscheider, and L. M. K. Vandersypen, Phys. Rev. Lett. 101, 226603 (2008).

${ }^{40}$ W. I. Wang, E. E. Mendez, T. S. Kuan, and L. Esaki, Appl. Phys. Lett. 47, 826 (1985).

${ }^{41}$ R. E. Williams, Modern GaAs Processing Methods, 2nd ed. (Artech, Boston, 1990), p. 226.

${ }^{42}$ J. R. Waldrop, Appl. Phys. Lett. 44, 1002 (1984); 47, 1301 (1985).

${ }^{43}$ S. M. Sze, Semiconductor Devices-Physics and Technology, 2nd ed. (Wiley \& Sons, New York, 2002), p. 228.

${ }^{44}$ D. H. Cobden, A. Savchenko, M. Pepper, N. K. Patel, D. A. Ritchie, J. E. F. Frost, and G. A. C. Jones, Phys. Rev. Lett. 69, 502 (1992).

${ }^{45}$ M. Pioro-Ladrière, J. H. Davies, A. R. Long, A. S. Sachrajda, L. Gaudreau, P. Zawadzki, J. Lapointe, J. Gupta, Z. Wasilewski, and S. Studenikin, Phys. Rev. B 72, 115331 (2005).

${ }^{46}$ G. D. Wilk, R. M. Wallace, and J. M. Anthony, J. Appl. Phys. 89, 5243 (2001).

${ }^{47}$ A. M. Sonnet, C. L. Hinkle, D. Heh, G. Bersuker, and E. M. Vogel, IEEE Trans. Electron. Devices 57, 2599 (2010).

${ }^{48}$ M. E. Gershenson, V. Podzorov, and A. F. Morpurgo, Rev. Mod. Phys. 78, 973 (2006).

${ }^{49}$ J. Veres, S. Ogier, G. Lloyd, and D. de Leeuw, Chem. Mater. 16, 4543 (2004).

${ }^{50}$ E. Buks, M. Heiblum, Y. Levinson, and H. Shtrikman, Semicond. Sci. Technol. 9, 2031 (1994).

${ }^{51}$ C. Berven, M. N. Wybourne, A. Ecker, and S. M. Goodnick, Phys. Rev. B 50, 14639 (1994).

${ }^{52}$ A. M. See, I. Pilgrim, B. C. Scannell, R. D. Montgomery, O. Klochan, A. M. Burke, M. Aagesen, P. E. Lindelof, I. Farrer, D. A. Ritchie, R. P. Taylor, A. R. Hamilton, and A. P. Micolich, Phys. Rev. Lett. 108, 196807 (2012).
${ }^{53}$ R. Murray, R. C. Newman, M. J. L. Sangster, R. B. Beall, J. J. Harris, P. J. Wright, J. Wagner, and M. Ramsteiner, J. Appl. Phys. 66, 2589 (1989).

${ }^{54}$ S. H. Kwok, R. Merlin, W. Q. Li, and P. K. Bhattacharya, J. Appl. Phys. 72, 285 (1992).

${ }^{55}$ M. J. Ashwin, M. R. Fahy, R. C. Newman, J. Wagner, D. A. Robbie, M. J. L. Sangster, I. Silier, E. Bauser, W. Braun, and K. Ploog, J. Appl. Phys. 76, 7839 (1994).

${ }^{56}$ S. A. McQuaid, R. C. Newman, M. Missous, and S. O'Hagan, J. Cryst. Growth 127, 515 (1993).

${ }^{57}$ R. C. Newman, M. J. Ashwin, M. R. Fahy, L. Hart, S. N. Holmes, C. Roberts, X. Zhang, and J. Wagner, Phys. Rev. B 54, 8769 (1996).

${ }^{58}$ M. J. Ashwin, R. C. Newman, and K. Muraki, J. Appl. Phys. 82, 137 (1997).

${ }^{59}$ C. Domke, Ph. Ebert, and K. Urban, Phys. Rev. B 57, 4482 (1998).

${ }^{60}$ S. S. Bose, B. Lee, M. H. Kim, G. E. Stillman, and W. I. Wang, J. Appl. Phys. 63, 743 (1988).

${ }^{61}$ Y. Nannichi, J.-F. Fan, H. Oigawa, and A. Koma, Jpn. J. Appl. Phys. 27, L2367 (1988).

${ }^{62}$ C. J. Sandroff, M. S. Hegde, and C. C. Chang, J. Vac. Sci. Technol. B 7, 841 (1989).

${ }^{63}$ R. S. Besser and C. R. Helms, J. Appl. Phys. 65, 4306 (1989).

${ }^{64}$ V. N. Bessolov, M. V. Lebedev, N. M. Binh, M. Freidrich, and D. R. T. Zahn, Semicond. Sci. Technol. 13, 611 (1998).

${ }^{65}$ D. Liu, T. Zhang, R. A. LaRue, J. S. Harris, Jr., and T. W. Sigmon, Appl. Phys. Lett. 53, 1059 (1988).

${ }^{66}$ N. Galbiati, E. Grilli, M. Guzzi, L. Brusaferri, L. Pavesi, and M. Henini, Semicond. Sci. Technol. 11, 1830 (1996).

${ }^{67}$ F. D. Auret and M. Nel, Appl. Phys. Lett. 48, 130 (1986).

${ }^{68}$ J.-H. Reemtsma, S. Kugler, K. Heime, W. Schlapp, and G. Weimann, J. Appl. Phys. 65, 2867 (1989).

${ }^{69}$ C. Radue, A. B. Conibear, and C. A. B. Ball, J. Appl. Phys. 84, 2030 (1998).

${ }^{70}$ L. H. Ho, W. R. Clarke, A. P. Micolich, R. Danneau, O. Klochan, M. Y. Simmons, A. R. Hamilton, M. Pepper, and D. A. Ritchie, Phys. Rev. B 77, 201402 (2008).

${ }^{71}$ L. H. Ho, A. P. Micolich, A. R. Hamilton, and O. P. Sushkov, Phys. Rev. B 80, 155412 (2009).

${ }^{72}$ V. Umansky, M. Heiblum, Y. Levinson, J. Smet, J. Nübler, and M. Dolev, J. Cryst. Growth 311, 1658 (2009).

${ }^{73}$ C. Rössler, T. Feil, P. Mensch, T. Ihn, K. Ensslin, D. Schuh, and W. Wegscheider, New. J. Phys. 12, 043007 (2010).

${ }^{74}$ P. M. Solomon, C. N. Knoedler, and S. L. Wright, IEEE Electron Device Lett. 5, 379 (1984).

${ }^{75}$ B. E. Kane, G. R. Facer, A. S. Dzurak, N. E. Lumpkin, R. G. Clark, L. N. Pfeiffer, and K. W. West, Appl. Phys. Lett. 72, 3506 (1998).

${ }^{76}$ W. R. Clarke, A. P. Micolich, A. R. Hamilton, M. Y. Simmons, K. Muraki, and Y. Hirayama, J. Appl. Phys. 99, 023707 (2006).

${ }^{77}$ O. Klochan, W. R. Clarke, R. Danneau, A. P. Micolich, L. H. Ho, A. R. Hamilton, K. Muraki, and Y. Hirayama, Appl. Phys. Lett. 89, 092105 (2006).

${ }^{78}$ O. Klochan, A. P. Micolich, L. H. Ho, A. R. Hamilton, K. Muraki, and Y. Hirayama, New J. Phys. 11, 043018 (2009).

${ }^{79}$ J. C. H. Chen, O. Klochan, A. P. Micolich, A. R. Hamilton, T. P. Martin, L. H. Ho, U. Zülicke, D. Reuter, and A. D. Wieck, New J. Phys. 12, 033043 (2010).

${ }^{80}$ O. Klochan, J. C. H. Chen, A. P. Micolich, A. R. Hamilton, K. Muraki, and Y. Hirayama, Appl. Phys. Lett. 96, 092103 (2010). 